\title{
Cosmological dynamics of non-minimally coupled scalar field system and its late time cosmic relevance
}

\author{
M. Sami \\ Kobayashi-Maskawa Institute for the Origin of Particles and the Universe, \\ Nagoya University, Nagoya 464-8602, Japan and \\ Centre for Theoretical Physics, Jamia Millia Islamia, New Delhi-110025, India \\ M. Shahalam \\ Centre for Theoretical Physics, Jamia Millia Islamia, New Delhi-110025, India \\ M. Skugoreva \\ Institute of Gravitation and Cosmology, Peoples Friendship \\ University of Russia, Moscow 117198, Russia \\ A. Toporensky \\ Sternberg Astronomical Institute, Moscow 119992, Russia
}

\begin{abstract}
We investigate the cosmological dynamics of non-minimally coupled scalar field system described by $F(\phi) R$ coupling with $F(\phi)=\left(1-\xi \phi^{N}\right) R(N \geq 2)$ and the field potential, $V(\phi)=V_{0} \phi^{n}$. We use a generic set of dynamical variables to bring out new asymptotic regimes of the underlying dynamics. However, our dynamical variables miss the most important fixed point - the de Sitter solution. We make use of the original form of system of equations to investigate the issues related to this important solution. In particular, we show that the de-Sitter solution which is a dynamical attractor of the system lies in the region of negative effective gravitational constant $G_{N}$ thereby leading to a ghost dominated universe in future and a transient quintessence(phantom) phase with $G_{N}>0$ around the present epoch 1 . We also carry out comparison of the model with other competing models of dark energy such as galileon modified gravity and others.
\end{abstract}

PACS numbers:

\section{INTRODUCTION}

Theories with a scalar field non-minimally coupled to gravity dubbed scalar tensor theories have been studied for decades. The first well-known example of non-minimal coupling a la Brans-Dicke theory, was proposed in 1961 with an aim to match Mach principle with General Relativity [1]. In this theory the gravitational constant is replaced by a scalar field $\phi$ entering into the action in a specific combination with Riemanian curvature as $\phi^{2} R$.

Followed by the Brans-Dicke proposal, other forms of scalar-tensor action were investigated, a well known example of a non-minimally coupled system is provided by $F(\phi) R$ coupling with $F=1-\xi \phi^{2}$. The cosmological dynamics of such a theory is rather rich and deserves attention. For a recent development in this direction, it is worth nothing that non-minimally coupled Higgs field due to a large coupling $\xi$ might give rise to a successful inflation [2] which is otherwise impossible.

The non-minimally coupled scalar field system due to novel features are of great interest to dark energy model building $3-11]$. For instance, non-minimal coupling might allow phantom crossing and may give rise to cosmological scaling solutions of interest to models of dark energy. Phantom scaling solutions are generic features of a non-minimally coupled system with $F=1-\xi \phi^{2}[13]$.

In recent years, methods of dynamical system theory have been extensively used in cosmology for obtaining a general picture of dynamics for many cosmological models including those with a scalar field and modified gravity. The advantage of this method is in having some kind of "machinery" for deriving asymptotic solution using a simple programmed algorithm. This requires introduction of new set of variables in which the initial system can be rewritten as a system of first-order equations. On the other hand, there is a danger of losing some important solution as well as inability for this scheme to find transient regimes which can also be important. Nevertheless, a classification of stable asymptotic regimes, given by this method may be useful for understanding the underlying dynamics. The success and

\footnotetext{
${ }^{1}$ However, as demonstrated by Starobinsky in 1981, the ghost dominated universe, if exists, can not be accessed from the Universe we live in, we shall say more about this important result in the last section.
} 
the limitation of the framework of dynamical systems applied to $f(R)$ theory can be found in Refs. 14] and [15].

We shall restrict our discussion to a polynomial functions $F$ of the form $F(\phi)=1-\xi \phi^{N}(N \geq 2)$ and power-law potentials for the scalar field, $V(\phi)=V_{0} \phi^{n}$ giving rise to generalization of models considered earlier[13, 15 17] (see also Ref. [18] on the related theme). The set of variables that we use can help in bringing out some generic features of the underlying dynamics and new asymptotic regimes missed in earlier studies. We should, however, note that the set of variables used in the present paper is not useful for the study of approximate Einstein regime in the system under consideration. For detailed description of this regime, other methods are required [19]. Secondly, our variables miss the existence of de Sitter solution and in order to investigate its existence and stability, we need to go back to initial variables to perform the analysis.

In this paper, we investigate cosmological dynamics of non-minimally coupled scalar field system with specific functional forms of coupling and field potential using a convenient set of dynamical variables. We shall focus on the asymptotic regimes of the solutions of interest and reveal the important features associated with the de Sitter solution. We also carry out comparison of the model with other competing models of dark energy, in particular, the galileon system which is generically non-minimally coupled.

\section{EQUATIONS OF MOTION}

Let us consider the scalar field system with non-minimal coupling in the form,

$$
S=\frac{1}{2} \int \sqrt{-g} d^{4} x\left[m_{P l}^{2} R-\left(g^{\mu \nu} \phi_{\mu} \phi_{\nu}+\xi R B(\phi)+2 V(\phi)\right)\right]+S_{M},
$$

where $m_{P l}^{2}=1 / 8 \pi G=1 / \kappa$, and $\xi$ is the dimensionless parameter and $S_{M}$ designates matter action.

In a homogenous isotropic Friedmann-Robertson-Walker universe with spatially flat metric,

$$
d s^{2}=-d t^{2}+a^{2}(t) d l^{2}
$$

the equations of motion which are obtained by varying the action (1) have the form,

$$
\begin{gathered}
H^{2}=\frac{\kappa}{3}\left(\frac{1}{2} \dot{\phi}^{2}+V(\phi)+3 \xi\left(H \dot{\phi} B^{\prime}(\phi)+H^{2} B(\phi)\right)+\rho\right), \\
R=\kappa\left(-\dot{\phi}^{2}+4 V(\phi)+3 \xi\left(3 H \dot{\phi} B^{\prime}(\phi)+\frac{R}{3} B(\phi)+\dot{\phi}^{2} B^{\prime \prime}(\phi)+\ddot{\phi} B^{\prime}(\phi)\right)+\rho(1-3 \omega)\right), \\
\ddot{\phi}+3 H \dot{\phi}+\frac{1}{2} \xi R B^{\prime}(\phi)+V^{\prime}(\phi)=0 .
\end{gathered}
$$

where $\rho$ and $p$ are the energy density and pressure of the ordinary matter with $p=\omega \rho$.

From the standard form of equations

$$
R_{i j}-\frac{1}{2} R g_{i j}=8 \pi G_{N}\left(T_{i j, \phi}+T_{i j, m}\right)=\kappa T_{i j}^{e f f},
$$

we read off the expression for $G_{N}$ as the effective Newtonian gravitational constant

$$
G_{N}=\frac{\kappa}{8 \pi(1-\kappa \xi B(\phi))}
$$

and we shall use this definition of $G_{N}$ hereafter. Ricci Scalar in chosen metric is given by, $R=6\left(2 H^{2}+\dot{H}\right)$. For convenience, we shall use the system of unites with $\kappa=6$.

Dividing (3), (4) (5) by $H^{2}(1-6 \xi B(\phi))$ and multiplying (5) by $\xi B^{\prime}(\phi)$, we obtain,

$$
1=\frac{\dot{\phi}^{2}}{H^{2}(1-6 \xi B(\phi))}+\frac{2 V(\phi)}{H^{2}(1-6 \xi B(\phi))}+\frac{6 \xi \dot{\phi} B^{\prime}(\phi)}{H(1-6 \xi B(\phi))}+\frac{2 \rho}{H^{2}(1-6 \xi B(\phi))},
$$




$$
\begin{aligned}
\frac{R}{H^{2}} & =-\frac{6 \dot{\phi}^{2}}{H^{2}(1-6 \xi B(\phi))}+\frac{24 V(\phi)}{H^{2}(1-6 \xi B(\phi))}+\frac{54 \xi \dot{\phi} B^{\prime}(\phi)}{H(1-6 \xi B(\phi))}+\frac{18 \xi \dot{\phi}^{2} B^{\prime \prime}(\phi)}{H^{2}(1-6 \xi B(\phi))} \\
& +\frac{18 \xi \ddot{\phi} B^{\prime}(\phi)}{H^{2}(1-6 \xi B(\phi))}+\frac{6 \rho(1-3 \omega)}{H^{2}(1-6 \xi B(\phi))}, \\
0= & \frac{\xi \ddot{\phi} B^{\prime}(\phi)}{H^{2}(1-6 \xi B(\phi))}+\frac{3 \xi \dot{\phi} B^{\prime}(\phi)}{H(1-6 \xi B(\phi))}+\frac{R}{H^{2}} \frac{\xi^{2} B^{\prime 2}(\phi)}{2(1-6 \xi B(\phi))}+\frac{V^{\prime}(\phi) \xi B^{\prime}(\phi)}{H^{2}(1-6 \xi B(\phi))} .
\end{aligned}
$$

In the discussion to follow, we shall use the dimensionless variables,

$$
\begin{aligned}
x & =\frac{\dot{\phi}^{2}}{H^{2}(1-6 \xi B(\phi))}, \quad y=\frac{2 V(\phi)}{H^{2}(1-6 \xi B(\phi))}, \quad z=\frac{6 \xi \dot{\phi} B^{\prime}(\phi)}{H(1-6 \xi B(\phi))}, \\
\Omega & =\frac{2 \rho}{H^{2}(1-6 \xi B(\phi))},
\end{aligned}
$$

and the dimensionless parameters that depend on the specific form of functions $B(\phi), V(\phi)$,

$$
A=\frac{B^{\prime}(\phi) \phi}{(1-6 \xi B(\phi))}, \quad b=\frac{B^{\prime \prime}(\phi) \phi}{B^{\prime}(\phi)}, \quad c=\frac{V^{\prime}(\phi) \phi}{V(\phi)} .
$$

Here $^{\prime}$ denotes derivative with respect to $\phi$.

Using the new variables and parameters, we can now cast the equations of motion (17), (8), (9) in the autonomous form,

$$
\begin{aligned}
& \frac{\dot{x}}{H}=\frac{d x}{d \ln a}=x^{\prime}=12 X \frac{x}{z}-2 x\left(\frac{Y}{6}-2\right)+x z, \\
& \frac{\dot{y}}{H}=\frac{d y}{d \ln a}=y^{\prime}=\frac{y z}{6 \xi} \frac{c}{A}-2 y\left(\frac{Y}{6}-2\right)+y z, \\
& \frac{\dot{z}}{H}=\frac{d z}{d \ln a}=z^{\prime}=6 X+\frac{z^{2}}{6 \xi} \frac{b}{A}-z\left(\frac{Y}{6}-2\right)+z^{2}, \\
& \frac{\dot{A}}{H}=\frac{d A}{d \ln a}=A^{\prime}=\frac{z}{6 \xi}(b+1)+A z, \\
& \frac{\dot{\Omega}}{H}=\frac{d \Omega}{d \ln a}=\Omega^{\prime}=\Omega\left(-3-3 \omega-2\left(\frac{Y}{6}-2\right)+z\right),
\end{aligned}
$$

where we have written equations for variables and parameters $x, y, z, A, \Omega$ and dropped $\dot{b} \& \dot{c}$ as we would be interested in the cases of $b, c=$ const (see Sec. III). While writing equation for $\Omega$, we used the continuity equation, $\dot{\rho}+3 H \rho(1+\omega)=0$. Secondly, the higher derivative terms involving $\dot{H}$ and $\ddot{\phi}$ in the equations of motion,

$$
X \equiv \frac{\xi \ddot{\phi} B^{\prime}(\phi)}{H^{2}(1-6 \xi B(\phi))}, \quad Y \equiv \frac{R}{H^{2}}
$$

and $\Omega$ get expressed through autonomous variables $x, y, z$ and are given by,

$$
\begin{aligned}
\Omega & =1-x-y-z \\
X(x, y, z) & =-\frac{z}{2}-\frac{z^{2}}{18\left(4 x+z^{2}\right)}\left(-6 x+12 y+\frac{z^{2} b}{2 \xi A}+\frac{y c}{\xi A}+3(1-x-y-z)(1-3 \omega)\right) \\
Y(x, y, z) & =\frac{4 x}{4 x+z^{2}}\left(-6 x+12 y+\frac{z^{2}}{4 \xi A}\left(2 b-\frac{y c}{x}\right)+3(1-x-y-z)(1-3 \omega)\right) .
\end{aligned}
$$


Substituting these expressions in the system (12), we finally obtain,

$$
\begin{aligned}
x^{\prime}= & 12 x\left(-\frac{1}{2}-\frac{z}{18\left(4 x+z^{2}\right)}\left(-6 x+12 y+\frac{z^{2} b}{2 \xi A}+\frac{y c}{\xi A}\right.\right. \\
& +3(1-x-y-z)(1-3 \omega)))-2 x\left(\frac{2 x}{3\left(4 x+z^{2}\right)}\right. \\
& \left(-6 x+12 y+\frac{z^{2}}{4 \xi A}\left(2 b-\frac{y c}{x}\right)+3(1-x-y-z)\right. \\
& (1-3 \omega))-2)+x z, \\
y^{\prime}= & \frac{y z}{6 \xi} \frac{c}{A}-2 y\left(\frac { 2 x } { 3 ( 4 x + z ^ { 2 } ) } \left(-6 x+12 y+\frac{z^{2}}{4 \xi A}\left(2 b-\frac{y c}{x}\right)\right.\right. \\
& +3(1-x-y-z)(1-3 \omega))-2)+y z, \\
z^{\prime}= & 6\left(-\frac{z}{2}-\frac{z^{2}}{18\left(4 x+z^{2}\right)}\left(-6 x+12 y+\frac{z^{2} b}{2 \xi A}+\frac{y c}{\xi A}\right.\right. \\
& +3(1-x-y-z)(1-3 \omega)))-z\left(\frac{2 x}{3\left(4 x+z^{2}\right)}\right. \\
A^{\prime}= & \frac{z}{6 \xi}(b+1)+A z . \\
& \left(-6 x+12 y+\frac{z^{2}}{4 \xi A}\left(2 b-\frac{y c}{x}\right)+3(1-x-y-z)\right. \\
& (1-3 \omega))-2)+\frac{z^{2}}{6 \xi} \frac{b}{A}+z^{2}, \\
& (b x)
\end{aligned}
$$

Let us note that $z$ appearing in the denominator in (12) cancels upside down after the substitution of (14) into (12) thereby telling us that $z=0$ is a regular point of the dynamical system.

In what follows, we shall investigate the autonomous system (12) for fixed points. We would specially be interested in stable solutions of interest to late time cosmic acceleration. For simplicity, we shall assume specific functional forms for functions $B(\phi)$ and $V(\phi)$.

\section{STATIONARY POINTS AND THEIR STABILITY $: B(\phi)=\phi^{N}, V(\phi)=V_{0} \phi^{n}$}

In this special case, $b=B^{\prime \prime}(\phi) \phi / B^{\prime}(\phi)=N-1$ and $c=V^{\prime}(\phi) \phi / V(\phi)=n$. Our autonomous system was written keeping this simple case in mind. We shall find stationary points equating to zero the left-hand sides of the system (12). Their stability will be established using the sign of the corresponding eigenvalues which we shall obtain numerically. We begin our discussion from the case, $N \neq 2(b \neq 1)$. In the case of $N=2$, a simple additional relation exists between $x$ and $z$, this case will be taken up separately in the Appendix. In the general case the form of this relation is more involved algebraically and its substitution into our system leads to cumbersome equations. We, therefore, prefer not to make use of it, we would rather check resulting solutions for consistency.

\section{A. The case of $b \neq 1(N \neq 2)$.}

After solving the system of algebraic equations obtained after equating the left hand sides of (12) to zero, we find the following stationary points:

1. Vacuum stationary line: $\quad x=1, y=0, z=0, A \in(-\infty,+\infty), \Omega=0$

This is a vacuum solution for which the corresponding eigenvalues are given by,

$$
\lambda_{1}=3-3 \omega, \quad \lambda_{2}=6, \quad \lambda_{3}=0, \quad \lambda_{4}=0 .
$$

As one of these eigenvalues is positive, the stationary line is unstable for any value of $\xi$ and $\omega$. 
The time dependence of scale factor can be found using the second relation in (13),

$$
Y_{\text {stat }}=\frac{R}{H^{2}}=6\left(2+\frac{\dot{H}}{H^{2}}\right)
$$

Since $Y_{\text {stat }}$ is constant, the above equation can easily be integrated to obtain the expression for $a(t)$

$$
a(t)=a_{0}\left|t-t_{0}\right|^{\frac{1}{2-\frac{Y_{s t a t}}{6}}}
$$

where $t_{0}$ is integration constant. Since $Y_{\text {stat }}=-6$ for the stationary point under consideration, we finally have

$$
a(t)=a_{0}\left|t-t_{0}\right|^{\frac{1}{3}}
$$

We note that at all stationary points either $t \rightarrow t_{0}$ or $t \rightarrow \infty$ which means that starting from anywhere in the phase space, the fixed points are approached in the said limits. And all functions of time $(a(t), \phi(t), H(t), \rho(t))$ either go to zero or infinity or become constants in this limit. For instance, the stationary line coordinate $A$ has the following limiting values

$$
\left\{\begin{array}{l}
0, \text { if } \phi(t) \rightarrow 0 \\
-\frac{N}{6 \xi}, \text { if } \phi(t) \rightarrow \infty \\
\frac{N \phi_{0}{ }^{N}}{1-6 \xi \phi_{0}{ }^{N}}, \text { if } \phi=\phi_{0}=\text { const }
\end{array}\right.
$$

1 ). In case of $\phi(t) \rightarrow 0$ and $A \rightarrow 0$, we find $\phi(t)$ using the coordinate $x$ of this stationary line. As $\left(1-6 \xi \phi^{N}\right) \rightarrow 1$ for $\phi(t) \rightarrow 0$, we have

$$
\begin{aligned}
& x_{\text {stat }} \rightarrow \frac{\dot{\phi}^{2}}{H^{2}}, \\
& \pm \dot{\phi}=\sqrt{x_{\text {stat }} H}= \pm \sqrt{x_{\text {stat }}} \frac{\dot{a}}{a} \\
& \phi(t)= \pm \frac{\sqrt{x_{\text {stat }}}}{2-\frac{Y_{\text {stat }}}{6}} \ln \left|\frac{t-t_{0}}{t^{\prime}-t_{0}^{\prime}}\right|
\end{aligned}
$$

where

$$
x_{\text {stat }}>0, \phi_{0}^{\prime}=\text { const, } t^{\prime}-t_{0}^{\prime}=\frac{a_{0}^{\prime 2-\frac{Y_{\text {stat }}}{6}}}{a_{0}^{2-\frac{Y_{\text {stat }}}{6}} e^{\phi_{0}^{\prime} \frac{2-\frac{Y_{s t a t}}{6}}{\sqrt{x_{s t a t}}}}}=\text { const. }
$$

For the stationary line under consideration, $x_{\text {stat }}=1, Y_{\text {stat }}=-6$ which gives

$$
\phi(t)= \pm \frac{1}{3} \ln \left|\frac{t-t_{0}}{t^{\prime}-t_{0}^{\prime}}\right|
$$

Thus $\phi(t) \rightarrow \pm \infty$ for $t \rightarrow t_{0}$ or $t \rightarrow \infty$. The obtained behavior of $\phi(t)$ contradicts the initial assumption that $\phi(t) \rightarrow 0$ and this case should therefore be discarded from the discussion.

2). In case $\phi(t) \rightarrow \infty$ and $A \rightarrow-\frac{N}{6 \xi}$, we can find $\phi(t)$ using the following combinations of stationary point coordinates

$$
\begin{aligned}
& \frac{x_{\text {stat }}}{A_{\text {stat }}}=\frac{\dot{\phi}^{2}}{H^{2} N \phi^{N}}=\frac{\dot{\phi}^{2} a^{2}}{N \phi^{N} \dot{a}^{2}}=\gamma_{\text {stat }}, \\
& \frac{\dot{\phi}^{2}}{\phi^{N}}=N \gamma_{\text {stat }}\left(\frac{\dot{a}}{a}\right)^{2} \\
& \text { where either } \gamma_{\text {stat }}>0, \phi^{N}>0 \text { or } \gamma_{\text {stat }}<0, \phi^{N}<0 \\
& \pm \frac{|\phi|^{1-\frac{N}{2}}}{\left(1-\frac{N}{2}\right)}-\phi_{0}^{\prime}=\sqrt{N\left|\gamma_{\text {stat }}\right|} \ln \left|\frac{a}{a_{0}^{\prime}}\right| \\
& |\phi(t)|^{\frac{2-N}{2}}= \pm \frac{3(2-N) \sqrt{N\left|\gamma_{\text {stat }}\right|}}{12-Y_{\text {stat }}} \ln \left|\frac{t-t_{0}}{t^{\prime}-t_{0}^{\prime}}\right|
\end{aligned}
$$


where

$$
t^{\prime}-t_{0}^{\prime}=\frac{a_{0}^{\prime 2-\frac{Y_{\text {stat }}}{6}}}{a_{0}^{2-\frac{Y_{\text {stat }}}{6}} e^{\phi_{0}^{\prime} \frac{2-\frac{Y_{\text {stat }}}{6}}{\sqrt{N\left|\gamma_{\text {stat }}\right|}}}}=\text { const }, \quad \gamma_{\text {stat }}=\text { const }, \quad \phi_{0}^{\prime}=\text { const },
$$

Since, for this stationary line, $\gamma_{\text {stat }}=x_{\text {stat }} / A_{\text {stat }}=1 / A=-6 \xi / N, Y_{\text {stat }}=-6$, we have

$$
|\phi(t)|^{\frac{2-N}{2}}= \pm \frac{(2-N) \sqrt{6|\xi|}}{6} \ln \left|\frac{t-t_{0}}{t^{\prime}-t_{0}^{\prime}}\right| .
$$

This solution exists for $0<N<2$.

3). Finally, if $\phi=\phi_{0}=$ const, $A=\frac{N \phi_{0}^{N}}{1-6 \xi \phi_{0} N}$, again by using the combination $\gamma_{\text {stat }}=\frac{x_{s t a t}}{A_{s t a t}}=\frac{1-6 \xi \phi_{0}{ }^{N}}{N \phi_{0}}$ and substituting it in the expression of $\phi(t)$ in eq.(20), we get the same behavior, namely,

$$
|\phi(t)|^{\frac{2-N}{2}}= \pm \frac{(2-N) \sqrt{\left|\frac{1-6 \xi \phi_{0} N}{\phi_{0}^{N}}\right|}}{6} \ln \left|\frac{t-t_{0}}{t^{\prime}-t_{0}^{\prime}}\right| .
$$

Thus $\phi(t) \rightarrow \pm \infty$ for $t \rightarrow t_{0}$ or $t \rightarrow \infty$ and contradicts the initial assumption that $\phi=\phi_{0}=$ const.

We therefore conclude that the stationary line reduces to a stationary point, $A=-\frac{N}{6 \xi}$.

We can now write the expression for the quantity $G_{N}$ using the coordinates of the stationary point, $A=B^{\prime}(\phi) \phi /(1-6 \xi B(\phi))=N \phi^{N} /\left(1-6 \xi \phi^{N}\right)=N /\left(\phi^{-N}-6 \xi\right)$, we find that $\phi^{N}=A /(N+6 \xi A)$ leading to $G_{N}=6 / 8 \pi\left(1-6 \xi \phi^{N}\right)=6(N+6 \xi A) / 8 \pi N$ which is positive when $\xi>0, A<-N / 6 \xi$ or $\xi<0, A>-N / 6 \xi$.

Since the stationary line reduces to a stationary point for which $A=-N / 6 \xi(\phi(t) \rightarrow \infty)$, it follows that $G_{N} \rightarrow 0$. We also note that the vacuum solution does not contain parameters, $\omega, \mathrm{n}$ and N.

2. $x=0, y=0, z=1, A=-\frac{b+1}{6 \xi}=-\frac{N}{6 \xi}, \Omega=0$

The eigenvalues are,

$$
\lambda_{1}=\frac{b-1}{b+1}=\frac{N-2}{N}, \quad \lambda_{2}=\frac{5+5 b-c}{b+1}=5-\frac{n}{N}, \quad \lambda_{3}=2-3 \omega, \quad \lambda_{4}=1 .
$$

This point is unstable because $\lambda_{4}$ is positive for any $\xi, \omega$. We can compute $a(t)$ by noting that $Y_{\text {stat }}=0$,

$$
a(t)=a_{0}\left|t-t_{0}\right|^{\frac{1}{2}}
$$

As for the expression for $\phi(t)$, we use the following combination of autonomous variables at the stationary point

$$
\begin{aligned}
& \frac{z_{\text {stat }}}{6 \xi A_{\text {stat }}}=\frac{\dot{\phi}}{\phi H}=\beta_{\text {stat }}, \\
& \phi(t)=\phi_{0}\left|t-t_{0}\right|^{\frac{\beta_{\text {stat }}}{2-\frac{Y_{\text {stat }}}{6}}} .
\end{aligned}
$$

where $\beta \equiv \frac{z}{6 \xi A}=\frac{\dot{\phi}}{\phi H}$ and we have made use of the fact that $\beta_{\text {stat }}=$ const. For the vacuum stationary point under consideration, $\beta_{\text {stat }}=\frac{z_{\text {stat }}}{6 \xi A_{\text {stat }}}=-\frac{1}{b+1}=-\frac{1}{N}$ which finally gives

$$
\phi(t)=\phi_{0}\left|t-t_{0}\right|^{-\frac{1}{2 N}}
$$

Consistency analysis shows that this solution exists for $N>2$ and $n<5 N$, so it never coexists with the solution discussed above under point 1 . They both correspond to the situation in which the scalar field potential is negligible. Power indexes in this solutions do not depend on $\xi, \omega, n$, however the function $\phi(t)$ contains dependence on $N$. We also note that since $A=-N / 6 \xi\left(\phi(t) \rightarrow \infty\right.$ for $\left.t \rightarrow t_{0}\right)$ in the present case, $G_{N}$ vanishes asymptotically at the stationary point. 
3. $x=0, y=0, z=-1+3 \omega, \quad A=-\frac{b+1}{6 \xi}=-\frac{N}{6 \xi}, \quad \Omega=2-3 \omega$

The corresponding eigenvalues are,

$$
\begin{aligned}
\lambda_{1}= & \frac{(1-b)(1-3 \omega)}{b+1}, \\
\lambda_{2}= & \frac{c(1-3 \omega)+3(b+1)(1+\omega)}{b+1}<0, \\
& \text { for } c>3(b+1), \omega_{0}<\omega<1, \\
\lambda_{3}= & -2+3 \omega<0, \text { for } \omega<\frac{2}{3}, \\
\lambda_{4}= & -1+3 \omega<0, \text { for } \omega<\frac{1}{3} .
\end{aligned}
$$

where $\omega_{0}=-\frac{3(b+1)+c}{3(b+1-c)}>\frac{1}{3}$ for $b>0, c>3(b+1)$.

As regions where $\lambda_{2}$ and $\lambda_{4}$ are negative do not intersect, this point is unstable (either a saddle or a repulsive node). For obtaining $a(t), \phi(t)$, we note that $Y_{\text {stat }}=0, \beta=\frac{1-3 \omega}{b+1}=\frac{1-3 \omega}{N}$. This tells us that,

$$
\begin{aligned}
& a(t)=a_{0}\left|t-t_{0}\right|^{\frac{1}{2}}, \\
& \phi(t)=\phi_{0}\left|t-t_{0}\right|^{\frac{1-3 \omega}{2 N}} .
\end{aligned}
$$

The time dependence of the matter density we obtain using its continuity equation

$$
\rho(t)=\rho_{0}\left|t-t_{0}\right|^{\frac{3(1+\omega)}{Y_{S t a t}-2}}=\rho_{0}\left|t-t_{0}\right|^{-\frac{3(1+\omega)}{2}}
$$

Power indexes of this solution do not contain parameters $\xi, n$ and they depend on $\omega, N$.

It follows from the definition of $\Omega$ that it must be positive to ensure that $\rho>0$ and $G_{N}>0$. Since $\Omega=2-3 \omega$ in the present case, we should have $\omega<2 / 3$ to avoid a pathological situation. We also note that since $A=-N / 6 \xi$ $(\phi(t) \rightarrow \infty)$, the effective Newtonian constant $G_{N}$ vanishes at the stationary point.

4.

$$
\begin{aligned}
x & =0, y=\frac{(b+1)(c(1-3 \omega)+3(b+1)(\omega+1))}{2 c^{2}}, \\
z & =\frac{3(b+1)(\omega+1)}{c}, A=-\frac{b+1}{6 \xi}=-\frac{N}{6 \xi}, \\
\Omega & =\frac{2 c^{2}-(b+1)(3(\omega+1)(b+c+1)+4 c)}{2 c^{2}}
\end{aligned}
$$

The corresponding eigenvalues are,

$$
\begin{aligned}
\lambda_{1} & =\frac{3(\omega+1)(b-1)}{c}, \\
\lambda_{2,3} & =\frac{\left.3(\omega+1)(b+1)^{2}+3 c(\omega-1)(b+1)\right)}{4 c(b+1)} \pm \frac{\sqrt{(b+1)\left(f_{1}(b, c) \omega+f_{2}(b, c)+9 \omega^{2} f_{3}(b, c)\right)}}{4 c(b+1)}, \\
\lambda_{4} & =\frac{3(\omega+1)(1+b)}{c}
\end{aligned}
$$

where

$$
\begin{aligned}
& f_{1}(b, c)=-210 c^{2}(b+1)+162\left(b^{3}+1\right)+192 b^{2} c+192 c+486 b(b+1)+384 b c+48 c^{3}, \\
& f_{2}(b, c)=81\left(1+b^{3}\right)+\left(243 b+17 c^{2}\right)(1+b)+174 c\left(1+b^{2}\right)-16 c^{3}+348 b c, \\
& f_{3}(b, c)=(1+b)(b+1+c)(9(b+1)-7 c) .
\end{aligned}
$$

and $\lambda_{1}, \lambda_{4}$ are positive for $-1<\omega \leqslant 1$ therefore this stationary point is unstable for any $\xi$. 
Analogous to previous points we get,

$$
\begin{aligned}
& Y=\frac{3(3(b+1)(\omega+1)+c(1-3 \omega))}{c}=\frac{3(3 N(\omega+1)+n(1-3 \omega))}{n}, \\
& \beta=-\frac{3(\omega+1)}{c}=-\frac{3(\omega+1)}{n} .
\end{aligned}
$$

then,

$$
\begin{aligned}
& a(t)=a_{0}\left|t-t_{0}\right|^{-\frac{2 n}{3(N-n)(\omega+1)}}, \\
& \phi(t)=\phi_{0}\left|t-t_{0}\right|^{\frac{2}{(N-n)}}, \\
& \rho(t)=\rho_{0}\left|t-t_{0}\right|^{\frac{2 n}{N-n}} .
\end{aligned}
$$

We note that power indices of this solution depend on $\omega, N, n$ and do not depend on the coupling constant $\xi$.

For $b+1=c(N=n)$, the power index of functions $a(t)$ and $\phi(t)$ is infinite and power-law solutions cease to exist. In this case the coordinates of the fixed point 4 are, $x=0, y=2, z=3(\omega+1), A=-\frac{b+1}{6 \xi}, \Omega=-(3 \omega+4)$. We note that $\Omega<0$ for $\omega \in[-1,1]$ and that from the definition of $\Omega$ in (10), it follows that either $\rho>0, G_{N}<0$ or $\rho<0$, $G_{N}>0$ which doesn't correspond to the real Universe. We also find in this case that $Y_{\text {stat }}=12, \beta=-\frac{3(1+\omega)}{N}$. This tells us that $\dot{H} / H^{2}=0$ and $\dot{\phi} / \phi=\beta H_{0}$ which is equivalent to

$$
\begin{aligned}
a(t) & =a_{0} e^{H_{0}\left(t-t_{0}\right)}, \\
\phi(t) & =\phi_{0} e^{\beta H_{0}\left(t-t_{0}\right)}=\phi_{0} e^{-\frac{3 H_{0}(1+\omega)\left(t-t_{0}\right)}{N}}, \\
\rho(t) & =\rho_{0} e^{-3 H_{0}(1+\omega)\left(t-t_{0}\right)} .
\end{aligned}
$$

As for the constants $H_{0}$ and $\rho_{0}$, we substitute (35) in definitions (10) of $y, \Omega$ taking into account that $N=n, y=2$ and $\Omega=-(3 \omega+4)$. We then have

$$
\begin{gathered}
y=\frac{2 V_{0} \phi^{n}}{H^{2}\left(1-6 \xi \phi^{N}\right)}=\frac{2 V_{0} \phi_{0}{ }^{N} e^{-3 H_{0}(1+\omega)\left(t-t_{0}\right)}}{H_{0}{ }^{2}\left(1-6 \xi \phi_{0}{ }^{N} e^{-3 H_{0}(1+\omega)\left(t-t_{0}\right)}\right)} \rightarrow-\frac{V_{0}}{3 H_{0}{ }^{2} \xi} \\
\Omega=\frac{2 \rho}{H^{2}\left(1-6 \xi \phi^{N}\right)}=\frac{2 \rho_{0} e^{-3 H_{0}(1+\omega)\left(t-t_{0}\right)}}{{H_{0}}^{2}\left(1-6 \xi \phi_{0}^{N} e^{-3 H_{0}(1+\omega)\left(t-t_{0}\right)}\right)} \rightarrow-\frac{\rho_{0}}{3 H_{0}{ }^{2} \phi_{0}{ }^{N} \xi}
\end{gathered}
$$

for $t \rightarrow t_{0}$ when $H_{0}>0$ (or for $t \rightarrow \infty$ when $H_{0}<0$ ).

Therefore,

$$
H_{0}^{2}=-\frac{V_{0}}{3 \xi y}=-\frac{V_{0}}{6 \xi}
$$

where $\xi<0, V_{0}>0$ or $\xi>0, V_{0}<0$, and

$$
\rho_{0}=-3 \Omega H_{0}{ }^{2} \phi_{0}{ }^{N} \xi=-\frac{V_{0} \phi_{0}{ }^{N}(3 \omega+4)}{2} .
$$

We next consider the behavior of quantities $T=T_{\phi}+T_{m}$ and $R=6 T_{\text {eff }}=8 \pi G_{N} T$ for the case $N=n$. Substituting the so obtained exponential solution, $a(t)=a_{0} e^{H_{0}\left(t-t_{0}\right)}, \phi(t)=\phi_{0} e^{-\frac{3 H_{0}(1+\omega)\left(t-t_{0}\right)}{N}}$ in (4), we find

$$
\begin{aligned}
T_{e f f} & =\frac{R}{6}=2 H_{0}{ }^{2}=\text { const } \\
G_{N} & =\frac{6}{8 \pi\left(1-6 \xi \phi_{0}^{N} e^{-3 H_{0}(1+\omega)\left(t-t_{0}\right)}\right)} \propto e^{3 H_{0}(1+\omega)\left(t-t_{0}\right)} \\
T & =T_{\phi}+T_{m} \\
& =e^{-3 H_{0}(1+\omega)\left(t-t_{0}\right)}\left(\phi_{0}{ }^{N}\left(4 V_{0}-27 \xi(1+\omega) H_{0}{ }^{2}+27 \xi(1+\omega)^{2} H_{0}{ }^{2}\right)+\rho_{0}(1-3 \omega)\right) \propto e^{-3 H_{0}(1+\omega)\left(t-t_{0}\right)}
\end{aligned}
$$


for $t \rightarrow t_{0}, H_{0}>0\left(\right.$ or $\left.t \rightarrow \infty, H_{0}<0\right)$.

It is therefore clear from the aforesaid that $G_{N}(t)$ grows as an exponent whereas $T(t)$ decreases with the same rate thereby leading a constant product $G_{N} T$. A remark about the exponentially expanding solution is in order. The solution though has features similar to de Sitter solution but does not really qualify for a true de Sitter as $G_{N}$ is not constant in this case. We shall say more about this point in the discussion of the vacuum solution to follow.

\section{Vacuum solution}

This solution corresponds to the following fixed point,

$$
x=0, y=\frac{5+5 b-c}{b+1+c}, z=-\frac{2(2+2 b-c)}{b+1+c}, \quad A=-\frac{b+1}{6 \xi}=-\frac{N}{6 \xi}, \quad \Omega=0 .
$$

The corresponding eigenvalues in this case are given by,

$$
\begin{aligned}
& \lambda_{1}=-\frac{2((b-1)(2(b+1)-c))}{(b+1)(b+1+c)}<0, \text { for } b>1 \text { and } c<2(b+1), \\
& \lambda_{2}=-\frac{2(2+2 b-c)}{b+1+c}<0, \text { for } c<2(b+1), \\
& \lambda_{3}=-\frac{5+5 b-c}{b+1}<0, \text { for } c<5(b+1) . \\
& \lambda_{4}=-\frac{(b+1)(3(b+1)(\omega+1)+c(7+3 \omega))-2 c^{2}}{(b+1)(b+1+c)}<0
\end{aligned}
$$

for $c<2(b+1)$ when $\omega \in[-1 ; 1]$,

The negativity of eigenvalues for this vacuum point show that it is stable for $c<2(b+1)$. Indeed, it is an attractive node for $b>1$ and $c<2(b+1)$ for any numerical values of $\xi$ and $\omega$, see Fig廿(a).

We note that,

$$
\begin{gathered}
Y=\frac{6 c(5+5 b-c)}{(b+1+c)(b+1)}=\frac{6 n(5 N-n)}{(N+n) N}, \\
\beta=\frac{2(2+2 b-c)}{(b+1+c)(b+1)}=\frac{2(2 N-n)}{(N+n) N}
\end{gathered}
$$

which gives rise to the following expressions for $a(t)$ and $\phi(t)$,

$$
\begin{aligned}
& a(t)=a_{0}\left|t-t_{0}\right|^{\frac{(N+n) N}{(2 N-n)(N-n)}}, \\
& \phi(t)=\phi_{0}\left|t-t_{0}\right|^{\frac{2}{N-n}}
\end{aligned}
$$

This solution contains parameters $N, n$ but is independent of $\xi$ and $\omega$. We note that power indexes in (40) are negative for $N<n<2 N$ and, therefore, $a(t), \phi(t)$ diverge leading to "Big Rip" singularity at $t=t_{0}$. This result is generalization for the analogous vacuum solution $a(t)=a_{0}\left|t-t_{0}\right|^{\frac{2(\xi(2+n)-1)}{\xi(n-2)(n-4)}}, \phi(t)=\phi_{0}\left|t-t_{0}\right|^{\frac{2}{2-n}}$ obtained in the case of $N=2[13,16]$.

Let us further investigate the nature of the fixed point. For $b+1=c(N=n)$ and also for $2(b+1)=c(2 N=n)$ power indexes of functions $a(t)$ and $\phi(t)$ diverge and power-law solutions should transform into exponential ones. Indeed, the coordinates of the fixed point in these cases are given by, $x=0, y=2, z=-1, A=-\frac{N}{6 \xi}, \Omega=0$ and $x=0, y=1, z=0, A=-\frac{N}{6 \xi}, \Omega=0$ respectively for $N=n$ and $2 N=n$. In the first case, $Y=12, \beta=\frac{1}{N}$ whereas $Y=12, \beta=0$ in the second. In case of $N=n$, we find that, $\frac{\dot{H}}{H^{2}}=0, H=H_{0}=$ const and

$$
\begin{aligned}
& a(t)=a_{0} e^{H_{0}\left(t-t_{0}\right)}, \\
& \phi(t)=\phi_{0} e^{\beta H_{0}\left(t-t_{0}\right)}=\phi_{0} e^{\frac{H_{0}\left(t-t_{0}\right)}{N}} .
\end{aligned}
$$

We can find out $H_{0}$, using the definition of the coordinate $y$ from (10),

$$
\begin{aligned}
y= & \frac{2 V_{0} \phi^{n}}{H^{2}\left(1-6 \xi \phi^{N}\right)}=\frac{2 V_{0} \phi_{0}{ }^{N} e^{H_{0}\left(t-t_{0}\right)}}{H_{0}^{2}\left(1-6 \xi \phi_{0}^{N} e^{H_{0}\left(t-t_{0}\right)}\right)} \\
& \rightarrow-\frac{V_{0}}{3 H_{0}^{2} \xi} \text { for } t \rightarrow \infty,\left(H_{0}>0\right) \text { or } t \rightarrow t_{0}\left(H_{0}<0\right) .
\end{aligned}
$$




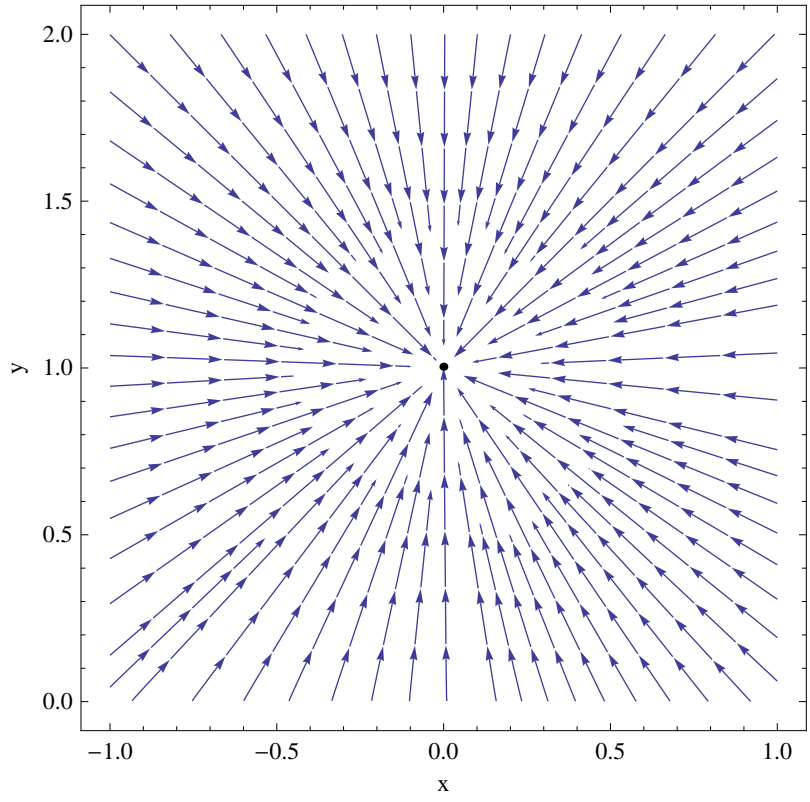

(a)

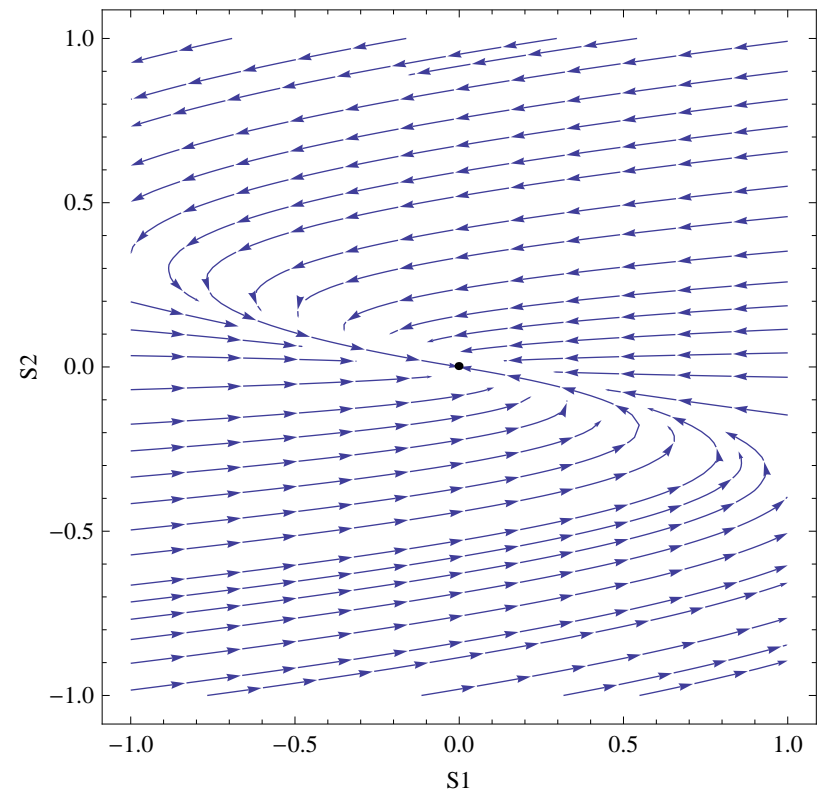

(b)

FIG. 1: The left panel (a) shows the Phase portrait for negative coupling constant $\xi=-1 / 2$ for the fixed point 5 , the fixed point is an attractive node.The right panel (b) displays the Phase portrait for positive coupling constant $\xi=0.3961$ $(N=2, n=7)$ for de sitter which is an attractive focus.

Thus, $H_{0}{ }^{2}=-\frac{V_{0}}{3 \xi y}=-\frac{V_{0}}{6 \xi}$ where $\xi<0, V_{0}>0$ or $\xi>0, V_{0}<0$.

In case of $n=2 N$, the fixed point under consideration does not lead to any physically admissible regime ( our numerical work shows that all trajectories in this case lead to oscillations near the minimally coupled field solution). It is interesting to note that in the absence of the standard curvature term in the action $\left(F(\phi) R=\phi^{N} R\right)$, a family of de Sitter solutions exists [20] for arbitrary values of field $\phi$. As for $n=N$, the solution corresponds to $\dot{H}=0$ and $w_{\text {eff }}=-1$ taken usually as the definition for de-Sitter solution tacitly assuming that Newtonian gravitational constant is a true constant of nature. In case, $\phi$ is constant, the constancy of $G_{N}$ is trivially satisfied. However, in the model under consideration, we have an interesting vacuum solution with $\dot{H}=0$ and an exponentially expanding solution for $\phi$ which corresponds to an exponentially decreasing/increasing (depending upon the sign of $H_{0}$ ) the effective Newtonian gravitational constant, $G_{N}$. The true de-Sitter corresponds to $\dot{H}=0$ and $\phi=$ const implying $G_{N}=$ const. Let us note that in this case, choosing $\phi_{0}=0$, one might think to obtain de Sitter solution but the latter implies $H_{0}=0$. Clearly, this solution does not qualify for a genuine de Sitter.

In fact, the true de-Sitter solution is not captured by the autonomous variables, we have used. In this case, the combination $4 x+z^{2}$ appearing in the denominator of autonomous system vanishes thereby telling us that autonomous variables used here are not suitable for the investigation of de-Sitter solution. We shall discuss this special case in a separate section to follow.

\section{INVESTIGATION OF de-SITTER SOLUTION: ITS EXISTENCE AND STABILITY}

As mentioned earlier, our autonomous system is not suitable for the study of de-Sitter solution corresponding to, $x=0, y=1, z=0, A=-\frac{n}{12 \xi}, \Omega=0$ as the combination, $4 x+z^{2}$ appearing in the denominator of dynamical system vanishes identically. Though the formalism of dynamical systems using autonomous variables is very powerful for the study of fixed points and their stability but it might miss some particular dynamical feature of the original system. In such a case we should go back to the original description to capture the same and this is what we shall do in the discussion to follow.

In this special, we go back to the original variables and make use of the system of equations (3), (4), (5). Substituting, $\dot{H}=\dot{\phi}=\ddot{\phi}=\rho=0$ and $R=12{H_{0}}^{2}$, for the de-Sitter solution in these equations we find, 


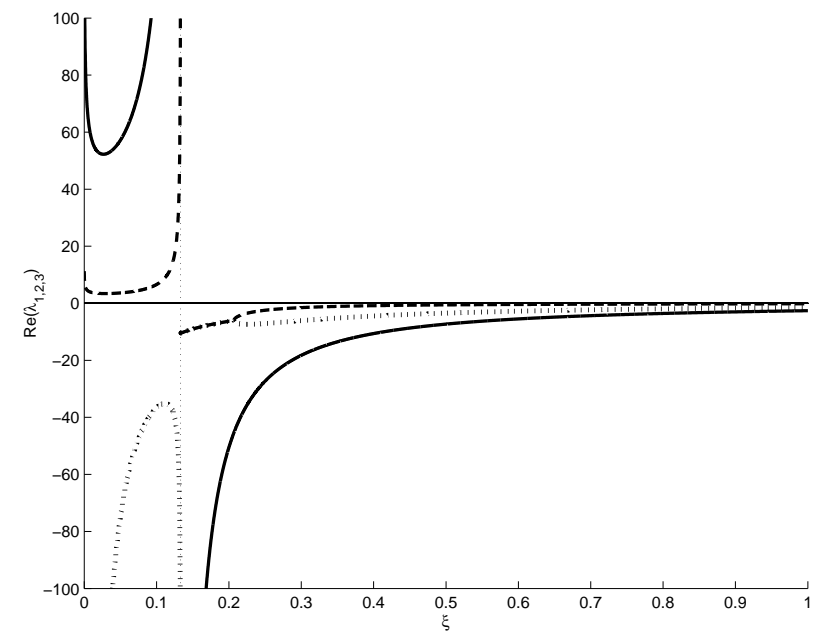

FIG. 2: This figure shows the real parts of eigen values for $N=2$ and $n=5$ versus $\xi$. The figure shows that the real part of two of the three eigen values is positive for $\xi<0.1333$ making the de Sitter solution unstable. Beyond the quoted value of $\xi$, real parts of all the eigen values remain negative. The two eigen values are complex conjugate of each other (their real parts coincide) in case of, $0.1333<\xi \leq 0.2068$ and we see only two curves. Beyond $\xi=0.2068$, the eigen value curve splits into two.

$$
H_{0}^{2}\left(1-6 \xi \phi_{0}{ }^{N}\right)=2 V_{0} \phi_{0}{ }^{n}, \quad 6 H_{0}^{2} \xi N \phi_{0}{ }^{N-1}+V_{0} n \phi_{0}{ }^{n-1}=0,
$$

which gives important information for solution under consideration,

$$
H_{0}^{2}=-\frac{V_{0} n \phi_{0}{ }^{n-N}}{6 \xi N}, \quad \phi_{0}{ }^{N}=\frac{n}{6 \xi(n-2 N)} .
$$

Clearly, this solution does not exist for $n>2 N$ in general for an even $n$. We also note that for de-Sitter solution , the effective Newtonian gravitational constant, $G_{N}=6 / 8 \pi(1-6 \xi B(\phi))=3(2 N-n) / 8 \pi N$ is positive only if $n<2 N$.

The system of equations (3), (44), (5) for $\rho=0$ which is of interest to us reduces to,

$$
\begin{aligned}
\dot{H}\left(1-6 \xi B+9 \xi^{2}{B^{\prime}}^{2}\right) & =3 \dot{\phi}^{2}\left(\xi B^{\prime \prime}-1\right)-3 \xi B^{\prime}\left(4 H \dot{\phi}+V^{\prime}+6 \xi B^{\prime} H^{2}\right) \\
\ddot{\phi}\left(1-6 \xi B+9 \xi^{2} B^{\prime 2}\right) & =-\left(3 H \dot{\phi}+V^{\prime}\right)(1-6 \xi B)-\xi B^{\prime}\left(-3 \dot{\phi}^{2}+12 V+9 \xi\left(3 H \dot{\phi} B^{\prime}+\dot{\phi}^{2} B^{\prime \prime}\right)\right) .
\end{aligned}
$$

In order to investigate the stability of the de-Sitter solution, we consider small perturbations $\mu \& \nu$ around this background: $H=H_{0}+\mu$ and $\phi=\phi_{0}+\nu$ in the dynamical system (45). Consequently, we find the evolution equations for perturbations

$$
\begin{aligned}
& \mu\left(36 \xi^{2} N^{2} H_{0}{\phi_{0}}^{2 N-2}\right)+\dot{\mu}\left(1-6 \xi \phi_{0}{ }^{N}+9 \xi^{2} N^{2}{\phi_{0}}^{2 N-2}\right)+\nu\left(3 \xi V_{0} N n(N+n-2) \phi_{0}{ }^{N+n-3}\right. \\
& \left.+36 \xi^{2} N^{2}(N-1) H_{0}{ }^{2} \phi_{0}{ }^{2 N-3}\right)+\dot{\nu}\left(12 \xi N H_{0} \phi_{0}{ }^{N-1}\right)=0 \\
& \quad \nu\left(V_{0} n(n-1) \phi_{0}{ }^{n-2}\left(1-6 \xi \phi_{0}{ }^{N}\right)-6 \xi V_{0} N n \phi_{0}{ }^{N+n-2}+12 \xi V_{0} N(N+n-1) \phi_{0}{ }^{N+n-2}\right) \\
& \quad+\dot{\nu}\left(3 H_{0}\left(1-6 \xi \phi_{0}{ }^{N}\right)+27 \xi^{2} N^{2} H_{0}{\phi_{0}}^{2 N-2}\right)+\ddot{\nu}\left(1-6 \xi \phi_{0}{ }^{N}+9 \xi^{2} N^{2} \phi_{0}{ }^{2 N-2}\right)=0 .
\end{aligned}
$$




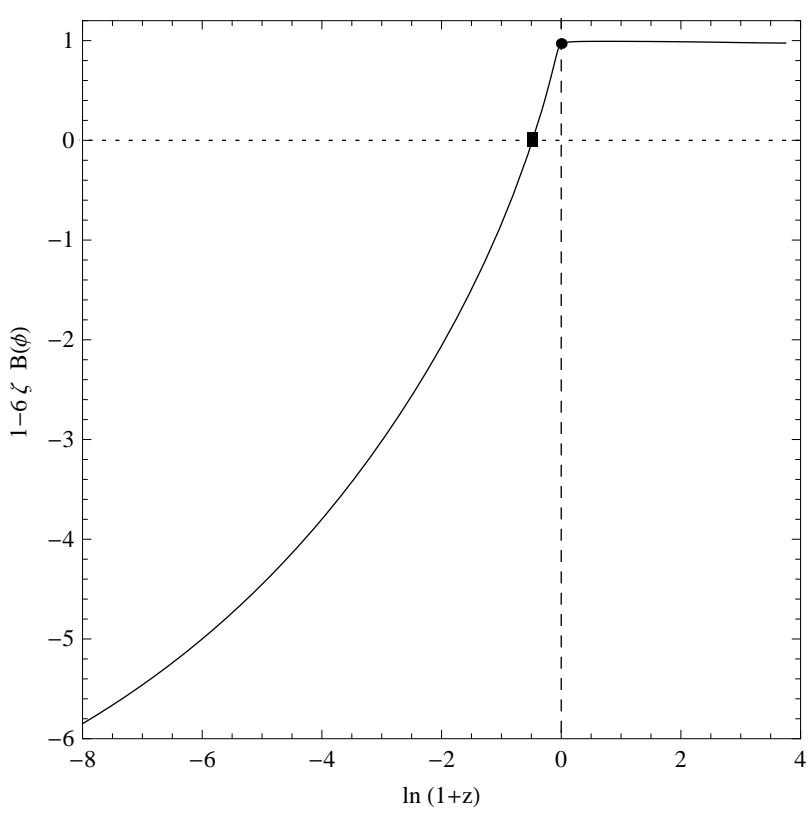

(a)

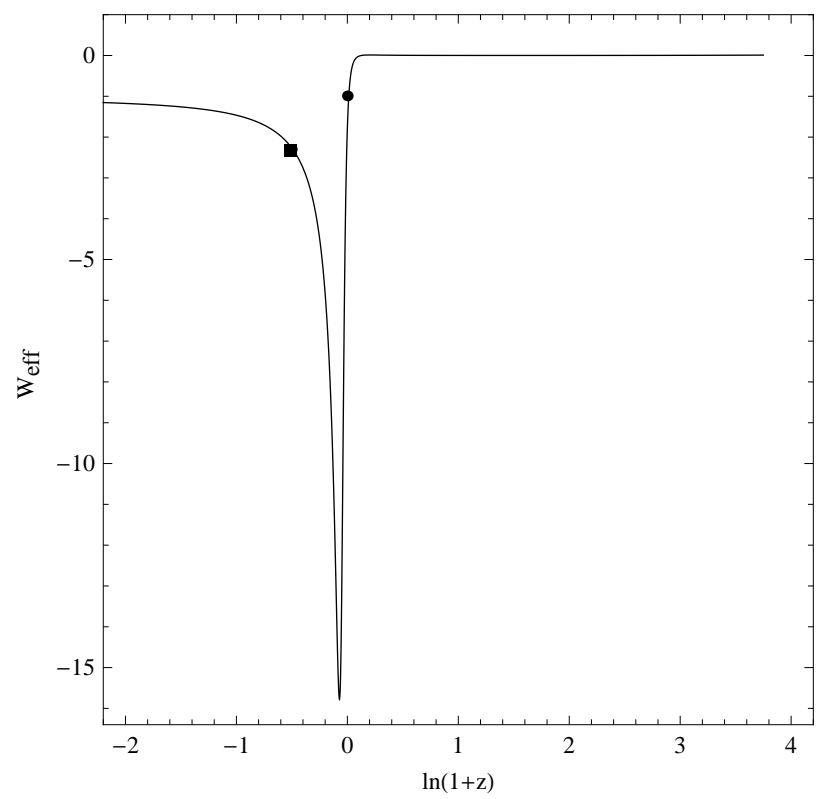

(b)

FIG. 3: The left panel (a) shows the evolution of $(1-6 \xi B(\phi))$ versus redshift $\mathrm{z}$, where $G_{N}=6 / 8 \pi(1-6 \xi B(\phi))$. The right panel (b) shows the evolution of $w_{\text {eff }}$ versus redshift $\mathrm{z}$. Both the figures correspond to the case of non-minimal coupling with $N=4, n=9, \xi=1 / 2$. We had chosen appropriate initial conditions, $H=2.034, \phi=-0.300, \dot{\phi}=-0.001$ and $\xi=0.5000$ (corresponding to $w_{m}=0$ initially) to obtain $w_{e f f} \simeq-1$ at the present epoch. The black dot in both the figures designates the present epoch which occurs in the regime of $G_{N}>0$, the effective Newtonian constant changes sign thereafter in future. The square marks the epoch where $G_{N}$ turns negative.

In order to cast equations (46), (47) in a simple form, we introduce the following notations

$$
\begin{aligned}
D & =36 \xi^{2} N^{2} H_{0}{\phi_{0}}^{2 N-2}, \\
E & =1-6 \xi \phi_{0}{ }^{N}+9 \xi^{2} N^{2}{\phi_{0}}^{2 N-2}, \\
F & =3 \xi V_{0} N n(N+n-2) \phi_{0}{ }^{N+n-3}+36 \xi^{2} N^{2}(N-1) H_{0}{ }^{2} \phi_{0}{ }^{2 N-3}, \\
G & =12 \xi N H_{0} \phi_{0}{ }^{N-1}, \\
D_{1} & =V_{0} n(n-1) \phi_{0}{ }^{n-2}\left(1-6 \xi \phi_{0}{ }^{N}\right)-6 \xi V_{0} N n \phi_{0}{ }^{N+n-2}+12 \xi V_{0} N(N+n-1) \phi_{0}{ }^{N+n-2}, \\
E_{1} & =3 H_{0}\left(1-6 \xi \phi_{0}{ }^{N}\right)+27 \xi^{2} N^{2} H_{0}{\phi_{0}}^{2 N-2}, \\
F_{1} & =1-6 \xi \phi_{0}{ }^{N}+9 \xi^{2} N^{2}{\phi_{0}}^{2 N-2}
\end{aligned}
$$

and make use of new variables $s_{1}=\mu, s_{2}=\nu, s_{3}=\dot{\nu}$. The system of equations (46) and (47) then acquires a simple form,

$$
\begin{aligned}
& D s_{1}+E \dot{\mu}+F s_{2}+G s_{3}=0, \\
& D_{1} s_{2}+E_{1} s_{3}+F_{1} \ddot{\nu}=0 .
\end{aligned}
$$

Taking derivative of $s_{1}, s_{2}, s_{3}$ with respect to time we get the system of equations,

$$
\left(\begin{array}{c}
\dot{s_{1}} \\
\dot{s_{2}} \\
\dot{s_{3}}
\end{array}\right)=\left(\begin{array}{ccc}
-\frac{D}{E} & -\frac{F}{E} & -\frac{G}{E} \\
0 & 0 & 1 \\
0 & -\frac{D_{1}}{F_{1}} & -\frac{E_{1}}{F_{1}}
\end{array}\right)\left(\begin{array}{l}
s_{1} \\
s_{2} \\
s_{3}
\end{array}\right)
$$

which we have studied numerically. The numerical investigation of this system for $b=N-1=1,2,3,4,5,6$ and $c=n=1,2,3,4,5,6,7,8,9$ shows that the de Sitter solution is stable when (we assume $V_{0}=1$ ) 


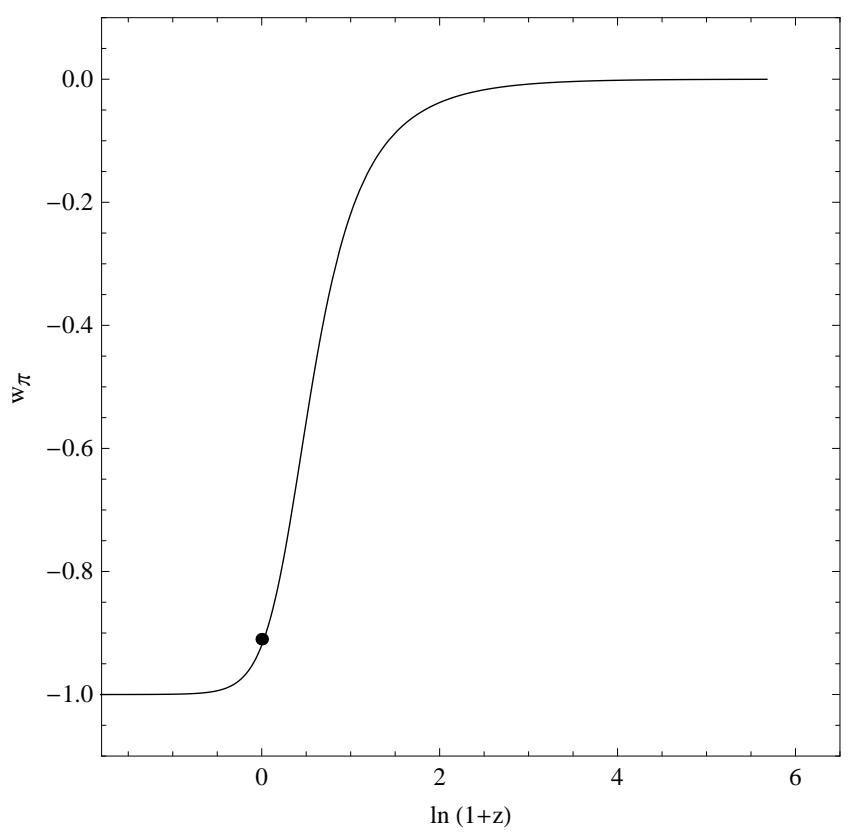

FIG. 4: This figure shows the evolution of the equation of state parameter $w_{\pi}$ versus redshift $\mathrm{z}$ for a scalar field $\pi$ in galileon model with Friedmann equation (54). The initial conditions were chosen carefully allowing us to be in the region of phase space where de Sitter is a stable fixed point 21]. The black dot on the curve designates the present epoch.

$2 N+1 \leqslant n=5,7,9, \ldots,(N=2,4,6, \ldots)$ and for positive values of the coupling, $\xi>\xi_{0}>0$, where $\xi_{0}$ depends on $b, c$. We hereby quote few cases which show how the nature of the fixed points crucially depends upon numerical values of the coupling $\xi$ :

- For $\mathrm{b}=1(\mathrm{~N}=2)$ and $\mathrm{c}=\mathrm{n}=5$, the fixed point is

saddle if $0<\xi \leq \xi_{0}, \xi_{0} \simeq 0.1333$,

attractive focus if $\xi_{0}<\xi \leq 0.2068$,

attractive node if $0.2069 \leq \xi \leq 1$;

- For $\mathrm{b}=1(\mathrm{~N}=2)$ and $\mathrm{c}=\mathrm{n}=7$, the fixed point is

saddle if $0<\xi \leq \xi_{0}, \xi_{0} \simeq 0.0952$,

attractive focus if $\xi_{0}<\xi \leq 0.3999$,

attractive node if $0.4000 \leq \xi \leq 1$;

- For $\mathrm{b}=1(\mathrm{~N}=2)$ and $\mathrm{c}=\mathrm{n}=9$, the fixed point is

saddle if $0<\xi \leq \xi_{0}, \xi_{0} \simeq 0.0740$,

attractive focus if $\xi_{0}<\xi \leq 1$;

- For $\mathrm{b}=3(\mathrm{~N}=4)$ and $\mathrm{c}=\mathrm{n}=9$, the fixed point is

saddle if $0<\xi<\xi_{0}, \xi_{0} \simeq 0.0009$,

attractive focus if $\xi_{0}<\xi \leq 0.0014$,

attractive node if $0.0015 \leq \xi \leq 1$.

The nature of eigen values of the perturbation matrix corresponding to eq. (51) crucially depends upon the numerical values of $b, c$ and $\xi$. For instance, the eigen values for the fourth point, $(N, n, \xi)=4,9,0.0012$ are, $-47990.1409,-2286.0463 \pm 1703.0817 i$ which shows that de Sitter solution is an attractive focus. We numerically investigated the region of stability of the solution, see Fig.2 We did not find stable de Sitter solution in the range of $n<2 N$ which has important implication for late time cosmology.

The stable de Sitter solution exists in the region where the effective Newtonian constant $G_{N}$ is negative which means that graviton is ghost thereby leading to instability. Before approaching the attractor, the system passes through a phantom phase and parameters in the theory can easily be adjusted such that we obtain the observed 


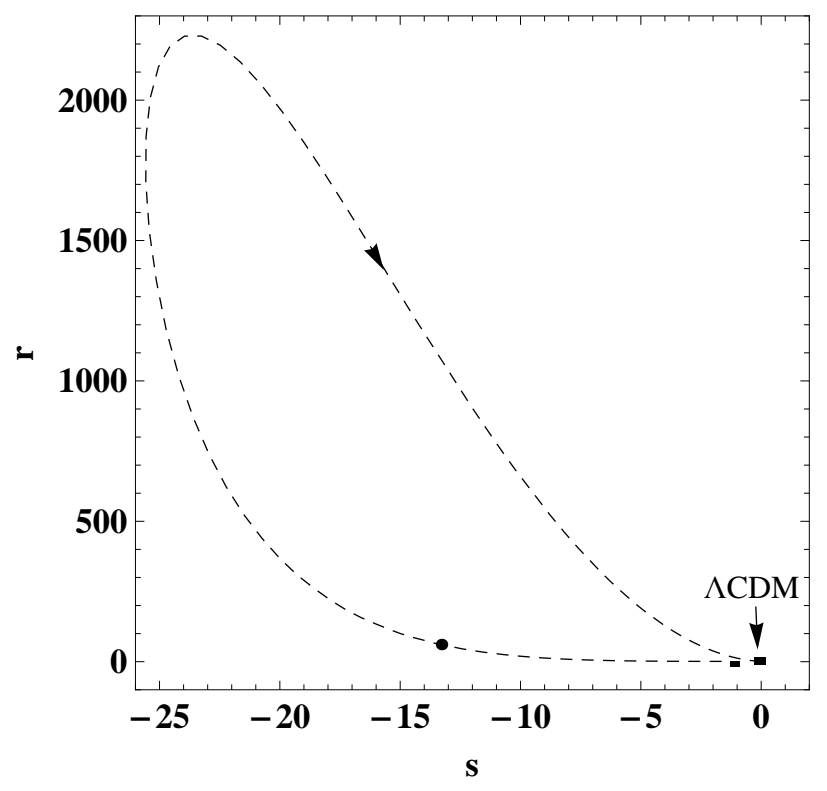

(a)

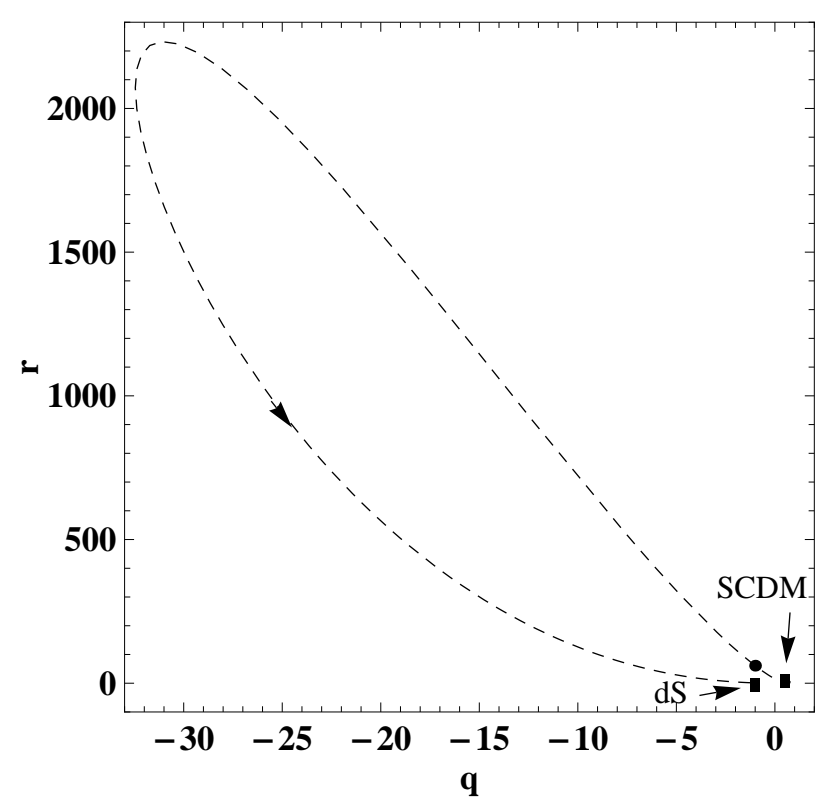

(b)

FIG. 5: In this figure, we plot the statefinder parameters for non-minimal model. The left panel (a) shows the time evolution of the statefinder pair $\{r, s\}$. The model converge to the fixed point $(r=1, s=0)$. The right panel (b) shows the time evolution of the statefinder pair $\{r, q\}$, the model diverge at the point $(r=1, q=0.5)$ which corresponds to matter dominated universe and converge to the point $(r=1, q=-1)$ which corresponds to de Sitter expansion. The dark dots on the curves show values $\left\{r_{0}, s_{0}\right\}$ (left) and $\left\{r_{0}, q_{0}\right\}$ at the moment when $w_{\text {eff }} \simeq-1$ from FIG. 3 (b) (right). Black squares show $(r=1, s=-1,0)($ left) and $(r=1, q=-1,0.5)$ (right).

value of equation of state parameter, $w_{e f f} \simeq-1$ at present with $G_{N}>0$ followed by a brief phantom phase before approaching the stable de Sitter fixed point ultimately pushing the ghost dominated regime to future (see Fig 3 ). It is also possible to set the phantom phase at the present epoch. We have carefully managed to shift the ghost regime to future by adjusting the parameters in the model. We should, however, admit that such a model of transient dark energy suffers from ugly fine tuning problem.

\section{COMPARISON WITH THE COMPETING MODELS}

There are several popular models of dark energy which can comply well with popular observational data sets. The list of models is rather large, we shall restrict to commonly used models: $\Lambda C D M$, Quintessence with tracking behavior, Chaplygin gas, DGP and recently discussed galileon model which generically belongs to the class of nonminimal coupled models.

One of the methods to distinguish between various models at the background level is provided by statefinder analysis. The Hubble parameter $H$ and the deceleration parameter $q$ constructed from the first and the second derivatives of the scale factor are often used to characterize a cosmological model. The statefinder method relies on the use of parameters constructed from third derivatives (and higher) of the scale factor [22, 23]. For instance, in the lowest order of this hierarchy, we have

$$
r=\frac{\dddot{a}}{a H^{3}}, \quad s=\frac{r-1}{3(q-1 / 2)}=1+w-\frac{1}{3} \frac{\dot{w}}{w H}
$$

which shows that $(r, s)=(1,0) \&(1,1)$ for $\Lambda C D M$ and matter dominated universe respectively. In what follows, we shall briefly describe the aforementioned dark energy models followed by their statefinder analysis. 


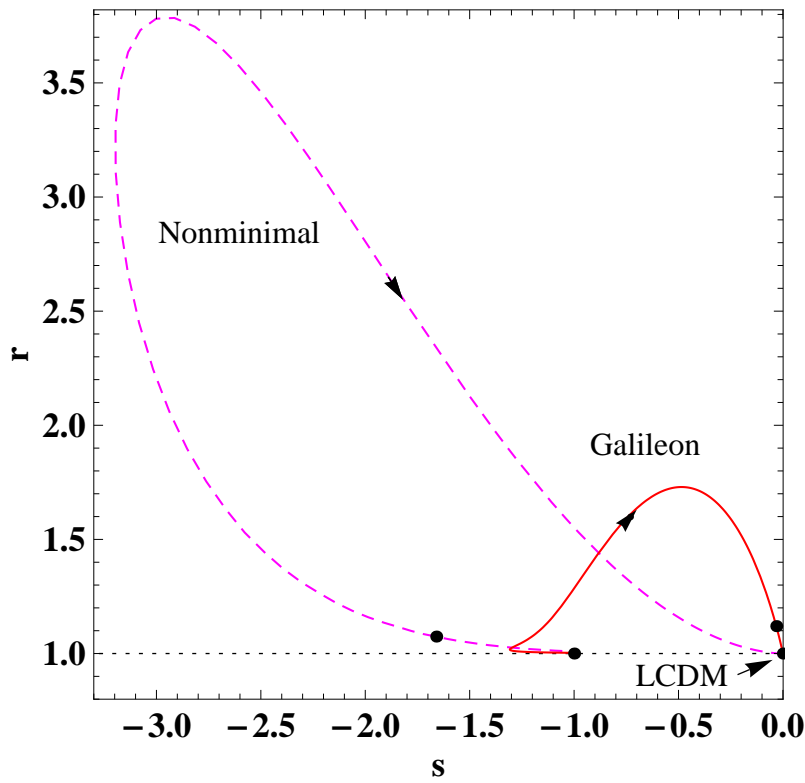

(a)

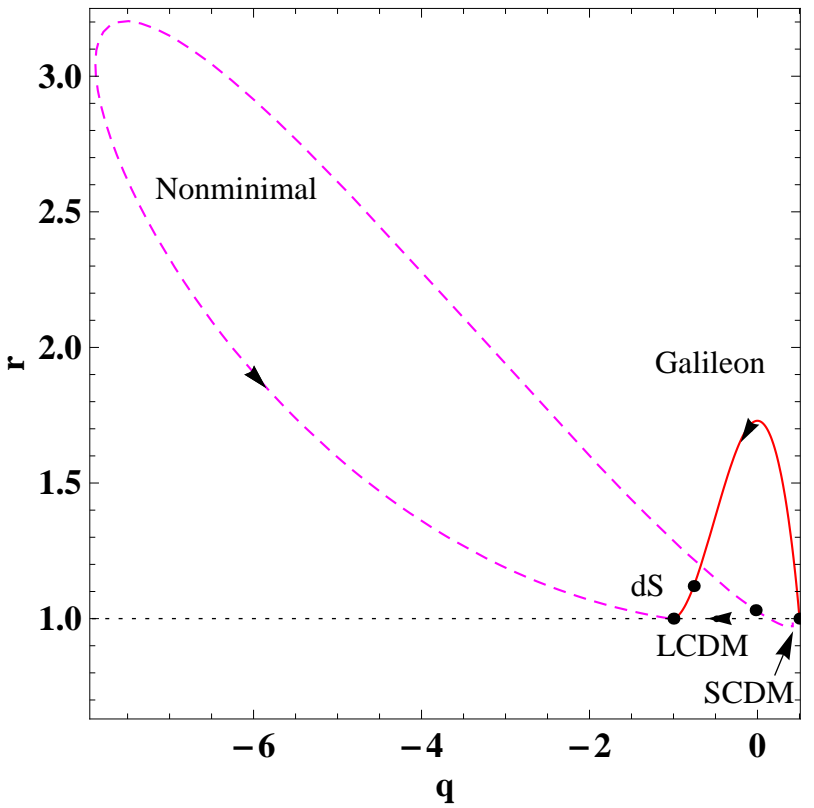

(b)

FIG. 6: (color online) The left panel (a) shows the time evolution of the statefinder pair $\{r, s\}$ for galileon (red) and Non-minimal (magenta) models. Both galileon and non-minimal models lie to the left of the $\Lambda$ CDM fixed point $(r=1, s=0)$. For both the models, $s$ increases to zero from -1 , whereas $r$ first increases from unity to a maximum value, then decreases to unity. Both models converge to the fixed point $(r=1, s=0)$ which corresponds to $\Lambda$ CDM.The right panel (b) shows the time evolution of the statefinder pair $\{r, q\}$ for galileon (red) and non-minimal (magenta) models. Both models diverge at the same point $(r=1, q=0.5)$ which corresponds to a matter dominated universe (SCDM) and converge to the same point $(r=1, q=-1)$ which corresponds to the de Sitter expansion(dS). The dark dots on the curves show current values $\left\{r_{0}, s_{0}\right\}$ (left) and $\left\{r_{0}, q_{0}\right\}$ (right) for different dark energy models. In all models, we have taken, $\Omega_{0 \mathrm{~m}}=0.3$ at the current epoch.

\section{DARK ENERGY MODELS}

\section{- Galileon model[21].}

Galileon is a massless scalar field, its Lagrangian apart from the kinetic term contains non-linear derivative terms responsible for switching off the modification to gravity locally. The theory is free from Ostrogradki ghosts despite the higher order derivative terms in the Lagrangian. The galileon action is of the form,

$$
\mathcal{S}=\int \mathrm{d}^{4} x \sqrt{-g}\left(\frac{R}{2}+c_{i} L^{(i)}\right)+\mathcal{S}_{m}\left[\psi_{m}, e^{2 \beta \pi} g_{\mu \nu}\right]
$$

where $\left\{c_{i}\right\}$ are constants, $\beta$ is coupling constant of field with matter and $L_{i}^{\prime} s$ [21] are Galileon Lagrangians. $L_{1}$ is linear in field and is often omitted assuming $c_{1}=0, L_{2}$ represents the standard kinetic terms, $L_{3}=\left(\partial_{\mu} \pi\right)^{2} \square \pi$ is the famous Vainshtein term in which three $\pi^{\prime} s$ participate; $L_{4}$ and $L_{5}$ are higher order Lagrangians. At least $L_{4}$ is needed to obtain dark energy solution in the model[21]. In this case, the evolution equations in a spatially flat background have the form [21]

$$
\begin{gathered}
3 H^{2}=\rho_{m}+\frac{c_{2}}{2} \dot{\pi}^{2}-3 c_{3} H \dot{\pi}^{3}+\frac{45}{2} c_{4} H^{2} \dot{\pi}^{4}, \\
2 \dot{H}+3 H^{2}=-\frac{c_{2}}{2} \dot{\pi}^{2}-c_{3} \dot{\pi}^{2} \ddot{\pi}+\frac{3}{2} c_{4} \dot{\pi}^{3}\left(3 H^{2} \dot{\pi}+2 \dot{H} \dot{\pi}+8 H \ddot{\pi}\right), \\
\beta \rho_{m}=-c_{2}(3 H \dot{\pi}+\ddot{\pi})+3 c_{3} \dot{\pi}\left(3 H^{2} \dot{\pi}+\dot{H} \dot{\pi}+2 H \ddot{\pi}\right)-18 c_{4} H \dot{\pi}^{2}\left(3 H^{2} \dot{\pi}+2 \dot{H} \dot{\pi}+3 H \ddot{\pi}\right)
\end{gathered}
$$




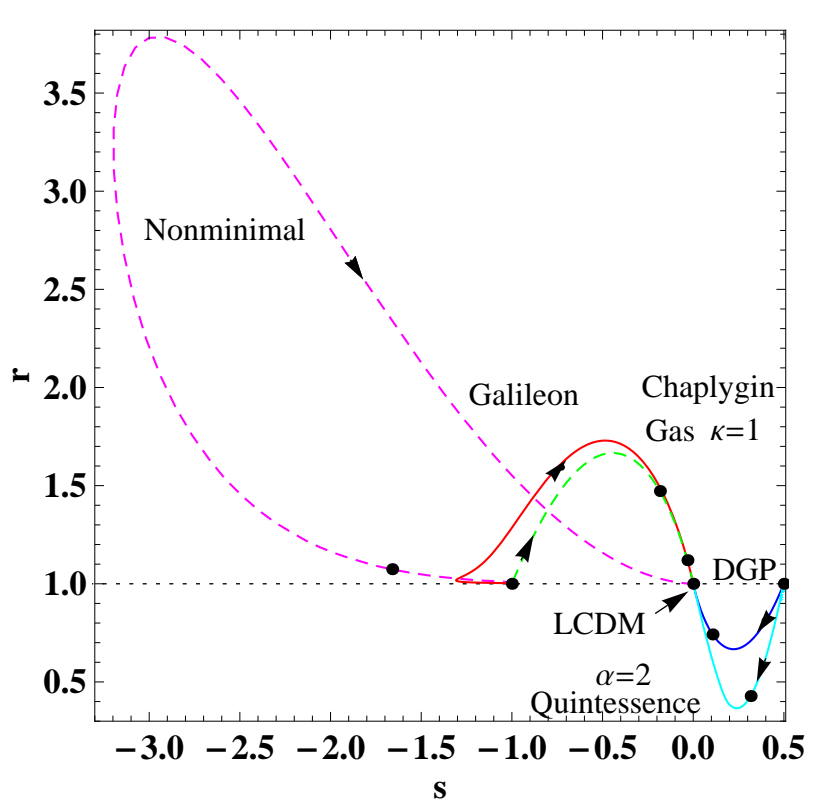

(a)

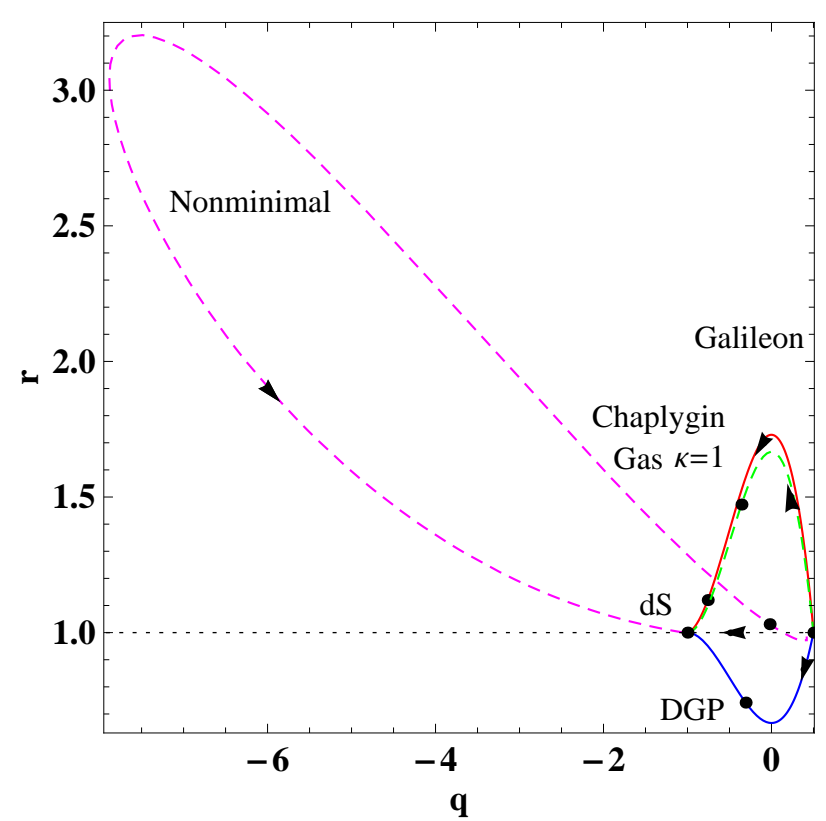

(b)

FIG. 7: (color online). The left panel (a) shows the time evolution of the statefinder pair $\{r, s\}$ for Galileon (red), Nonminimal (magenta), DGP (blue), quintessence (Cyan) and Chaplygin gas (green) models. All models converge to the fixed point $(r=1, s=0)$ which corresponds to $\Lambda$ CDM.The right panel (b) shows the time evolution of the statefinder pair $\{r, q\}$ for Galileon (red), nonminimal (magenta), DGP (blue) and chaplygin gas (green) models. All models diverge at the same point $(r=1, q=0.5)$ which corresponds to a matter dominated universe (SCDM), and converge to the same point $(r=1, q=-1)$ which corresponds to the de Sitter expansion(dS). The dark dots on the curves show current values $\left\{r_{0}, s_{0}\right\}$ (left) and $\left\{r_{0}, q_{0}\right\}$ (right) for different dark energy models. In all models, $\Omega_{0 \mathrm{~m}}=0.3$ at the current epoch.

The energy density, pressure for the scalar field $\pi$ and the Friedmann equation are,

$$
\begin{aligned}
\rho_{\pi} & =\frac{c_{2}}{2} \dot{\pi}^{2}-3 c_{3} H \dot{\pi}^{3}+\frac{45}{2} c_{4} H^{2} \dot{\pi}^{4}, \\
p_{\pi} & =\frac{c_{2}}{2} \dot{\pi}^{2}+c_{3} \dot{\pi}^{2} \ddot{\pi}-\frac{3}{2} c_{4} \dot{\pi}^{3}\left(3 H^{2} \dot{\pi}+2 \dot{H} \dot{\pi}+8 H \ddot{\pi}\right) \\
H^{2} & =\frac{8 \pi G}{3}\left[\rho_{0 m}(1+z)^{3}+\rho_{\pi}\right] ; \quad w_{\pi}=\frac{p_{\pi}}{\rho_{\pi}}
\end{aligned}
$$

As demonstrated in Ref. 21], there is a region in phase space which necessarily contains a stable de Sitter solution. In Fig团 we have plotted equation of state parameter $w_{\pi}$ (with favorable initial conditions) which shows that the latter ultimately approaches its de Sitter value.

\section{- Dark energy with a constant equation of state[5].}

The Hubble parameter is given by,

$$
H(z)=H_{0}\left[\Omega_{0 \mathrm{~m}}(1+z)^{3}+\Omega_{\mathrm{DE}}(1+z)^{3(1+w)}\right]^{1 / 2}
$$

where $(\Lambda \mathrm{CDM})$ corresponds to $w=-1$ and for Quiessence [22] $w=$ constant $\neq-1$.

- Non-minimally coupled scalar field [13]. The energy density and pressure in this case are given by,

$$
\begin{aligned}
\rho_{\phi} & =\frac{1}{2} \dot{\phi}^{2}+V(\phi)+3 \xi\left(H \dot{\phi} B^{\prime}(\phi)+H^{2} B(\phi)\right) \\
p_{\phi} & =\frac{1}{2} \dot{\phi}^{2}-V(\phi)-\xi\left(2 H \dot{\phi} B^{\prime}(\phi)+\dot{\phi}^{2} B^{\prime \prime}(\phi)+\ddot{\phi} B^{\prime}(\phi)+\left(2 \dot{H}+3 H^{2}\right) B(\phi)\right) .
\end{aligned}
$$


The Hubble parameter for this model has the form,

$$
H^{2}=\frac{8 \pi G}{3}\left[\rho_{0 m}(1+z)^{3}+\rho_{\phi}\right] ; \quad w_{\phi}=\frac{p_{\phi}}{\rho_{\phi}}
$$

\section{- Quintessence [5, 7].}

In this case, there is a variety of models which can lead to desired cosmic evolution with a carefully chosen field potential. All the quintessence models can be classified into two groups: Thawing and Freezing or tracker models. In case of thawing, cosmic evolution crucially depends upon initial conditions where as the tracker dynamics is free from the choice of initial conditions and we shall focus on the latter. Bearing that in mind, we have the expression for Hubble parameter,

$$
H^{2}=\frac{8 \pi G}{3}\left[\rho_{0 m}(1+z)^{3}+\rho_{\phi}\right] ; \quad V(\phi) \propto \phi^{-\alpha}, \alpha \geq 1
$$

- Chaplygin gas [29].

$$
H(z)=H_{0}\left[\Omega_{0 \mathrm{~m}}(1+z)^{3}+\frac{\Omega_{0 \mathrm{~m}}}{\kappa} \sqrt{\frac{A}{B}+(1+z)^{6}}\right]^{1 / 2} ; \kappa=\rho_{0 \mathrm{~m}} / \sqrt{B}, \quad A=B\left\{\kappa^{2}\left(\frac{1-\Omega_{0 \mathrm{~m}}}{\Omega_{0 \mathrm{~m}}}\right)^{2}-1\right\} .
$$

- The Dvali, Gabadadze , Porrati (DGP) model [30]:

$$
\frac{H(z)}{H_{0}}=\left[\left(\frac{1-\Omega_{0 \mathrm{~m}}}{2}\right)+\sqrt{\Omega_{0 \mathrm{~m}}(1+z)^{3}+\left(\frac{1-\Omega_{0 \mathrm{~m}}}{2}\right)^{2}}\right] .
$$

In Fig 5 we have shown the time evolution of the statefinder pairs $\{r, s\}$ and $\{r, q\}$ for non-minimally coupled system. The model converge to the fixed point $(r=1, s=0)$ which corresponds to $\Lambda$ CDM. The right panel (b) shows the time evolution of the statefinder pair $\{r, q\}$, the model diverge at the point $(r=1, q=0.5)$ which corresponds to a matter dominated universe (SCDM), and converge to the point $(r=1, q=-1)$ which corresponds to the de Sitter expansion(dS).

For comparision with other models, we have displayed our model along with other competing models in Figs. (6) \& (77). Since, to the best of our knowledge, statefinder analysis for galileon is not done elsewhere, we have in Fig. (6) displayed $(r, s)$ trajectories for non-minimally coupled scalar field system and galileon field which is generically nonminimally coupled system. Fig:7 displays statefinder trajectories for non-minimally coupled scalar field along with other popular models of dark energy including the galileon field. The figure shows that all models diverge from the same point $(r=1, q=0.5)$ which corresponds to a matter dominated universe (SCDM) and converge to the point $(r=1, q=-1)$ which represents de Sitter universe(dS). Fig 6 shows how clearly the two non-minimally coupled systems get distinguished on the $(\mathrm{r}, \mathrm{s})$ \& $(\mathrm{r}, \mathrm{q})$ planes. In case of galileon, we focussed on the sector of the model which contains stable de Sitter fixed points. Fig:7allows to distinguish these two models from other popular models of dark energy. It may be noted that we have re-scaled appropriately the statefinder variables of the model under consideration in order to strike comparison with other models. It is interesting to note that the behavior of statefinder pairs for galileon and Non-minimally coupled scalar field systems resembles with that of the braneworld models studied in Ref.[32].

\section{CONCLUSION}

In this paper, we have revisited cosmological dynamics of non-minimally coupled scalar field system. We presented detailed investigation of dynamics of the underlying system in case of $F(\phi) R=(1-\xi B(\phi)) R$ coupling with $B(\phi)=\phi^{N}$ and $V(\phi)=V_{0} \phi^{n}(N \geq 2)$ using a convenient set of autonomous variables. We studied asymptotic regimes of solutions in the model. In case of the vacuum solution, we found a very interesting solution for which $\dot{H}=0\left(w_{\text {eff }}=-1\right)$ and the scalar field $\phi$ increasing exponentially giving rise to exponentially decreasing $G_{N}$ - effective Newtonian gravitational constant. Such a solution does not qualify for de Sitter for which $G_{N}$ should be held constant.

The autonomous variables used in this paper though convenient in general but miss certain important features of the dynamics. The description fails to capture the de Sitter solution as the combination of variables $4 x+z^{2}$ appearing in the denominator of the autonomous system vanishes identically in this case. The investigation of this solution took us 
back to the original variable in the evolution equations. We found that in case of de Sitter solution, $G_{N}>0$ provided that $n<2 N$. On the other hand, our numerical investigations showed that the solution under consideration is stable only for $n \geq 2 N+1$ (we checked for lower values of $N \geq 2$ ). For initial conditions of matter dominated universe, the system enters the phase of acceleration consistent with observation at present followed by a brief phantom phase thereafter which continues till de Sitter is reached, see Fig (b) (which is an attractive focus of the dynamics, see Fig[1(b)). During the phantom phase $G_{N}$ changes sign from positive to negative thereby making the universe ghost dominated in future, see Fig 3 . It is possible to set parameters in the model such that phantom phase occurs at present epoch corresponding to $G_{N}>0$ compilable with observed values of the equation of state and fractional density parameter pushing the ghost dominated phase to future which no body has yet seen. Incidentally, similar features of equation of state are shared by the braneworld model discussed in Ref. 33.

An important remark about the ghost dominated universe with $G_{N}<0$ is in order. It was demonstrated by Starobinsky in 1981 that any attempt of crossing over to the regime with $G_{N}<0$ makes the Friedmann universe unstable at the transition point. Any tiny perturbation over homogeneity and isotropy grows large at the point of transition. Taking into account the quantum effects in the scalar sector does not affect this result ${ }^{1}$. The ghost dominated universe with $G_{N}<0$, if exists, will be separated from our real universe by a region of large curvature of the order of Planck value. Thus it is impossible to cross the point where the effective gravitational constant changes sign due to the formation of generic anisotropic curvature singularity at this point [34].

The de Sitter solution for the case with $N=2$ also shares the aforementioned features. In other cases, we have shown that the solutions obtained earlier are continued for values of $N>2$. We have investigated in detail the asymptotic regimes of these solutions in all cases including the one corresponding to $N=2$

We have shown that the non-minimally coupled scalar field system can account for late time cosmic acceleration. We should, however, emphasize that dark energy, in certain sense, in this scenario appears as a transient phenomenon which involves extra fine tuning. The transient phase is followed by a stable de Sitter point which lies in the regime of negative value of effective gravitational constant and can not be reached from the Universe we live in, a curvature singularity separates us from the de Sitter fixed point. In view of the phase space evolution, all the trajectories, at the present epoch, nearly converge to the one with equation of state parameter around -1 . The small change of initial conditions slightly changes the present value of equation of state parameter of dark energy. Tuning the initial conditions we can achieve phantom or non-phantom dark energy consistent with observations on late time cosmic acceleration.

Last but not least, we used statefinder analysis to compare the model with other rival models of dark energy, in particular, with galileon model which generically a non-minimally coupled system. The model under consideration is clearly distinguished on the $(r, s)$ and $(r, q)$ planes from other competing models of dark energy. We focussed our attention on sectors which contain stable de Sitter solution in case of the model under consideration as well as the galileon modified theory of gravity.

A final remark about the ghost dominated evolution in the model is in order. In the framework of the simple set up of non-minimal scheme discussed here, there exists no consistent de Sitter solution such that $G_{N}$ remains positive throughout the evolution. It would really be interesting to explore generic functional forms of the coupling function and the field potential to check for a well behaved de Sitter solution.

\section{Acknowledgments}

We are indebted to A. starobinsky for his kind comment on the impossibility of crossing over to ghost dominated universe. We thank V. Sahni, E. Saridakis and S. Yu. Svernov for useful comments. This work was supported in part by the FTsP "Nauchnie i nauchno-pedagogicheskie kadry innovatsionnoy Rossii" for the years 2009-2013". MSA acknowledge the financial support provided by the DST, Government of India, through the research project N0. SR/S2/HEP-002/2008. He is thankful to Amna Ali and Wali Hossain for fruitful discussions. MS thanks S. Nojiri and K. Bamba for useful discussions. M.S. is supported by the JSPS Invitation Fellowship for Research in Japan (Long-Term) \# L12422 and also by the Department of Science and Technology, India. MS thanks Kobayashi-Maskawa Institute for the Origin of Particles and the Universe for hospitality. AT is supported by RFBR grant no 11-02-00643 of the Russian Ministry of Science and Technology.

1 Though the higher order curvature corrections to Einstein-Hilbert action motivated by Starobinsky in different context could help to avoid the catastrophe and land the theory ghost free. 


\section{APPENDIX}

A. The case of $b=1(N=2)$.

As mentioned before, this case was considered earlier in other references. However, discussion of asymptotic regimes was missing and important issues related to the structure of de Sitter solution were not discussed. Thus we have retained this subsection in the appendix which is also useful for comparison of our results with $b=1(N=2)$ case.

As mentioned above, there exists a simple relation between $x$ and $z$ for $N=2$. Indeed we have,

$$
x=\frac{\dot{\phi}^{2}}{H^{2}(1-6 \xi B(\phi))}=\frac{\dot{\phi}^{2}}{H^{2}(1-6 \xi B(\phi))} \frac{\left(6 \xi B^{\prime}(\phi)\right)^{2}}{\left(6 \xi B^{\prime}(\phi)\right)^{2}} \frac{\phi(1-6 \xi B(\phi))}{\phi(1-6 \xi B(\phi))}=\frac{z^{2}}{72 \xi^{2} A}
$$

Now we substitute (66) and $b=1$ in (14),

$$
\begin{aligned}
\Omega= & 1-\frac{z^{2}}{72 \xi^{2} A}-y-z \\
X= & -\frac{z}{2}-\frac{1}{1+18 \xi^{2} A}\left(\frac{z^{2}}{12}(6 \xi-1)+y \xi(12 \xi A+c)\right. \\
& \left.+3 \xi^{2} A\left(1-\frac{z^{2}}{72 \xi^{2} A}-y-z\right)(1-3 \omega)\right), \\
Y= & \frac{1}{1+18 \xi^{2} A}\left(\frac{z^{2}}{12 \xi^{2} A}(6 \xi-1)+6 y\right. \\
& \left.(2-3 c \xi)+3\left(1-\frac{z^{2}}{72 \xi^{2} A}-y-z\right)(1-3 \omega)\right) .
\end{aligned}
$$

Excluding from (12) $x, \Omega, X, Y$ using (67), we obtain following system for $b=1(N=2)$

$$
\begin{aligned}
y^{\prime}= & \frac{y z}{6 \xi} \frac{c}{A}-2 y\left(\frac{1}{6\left(1+18 \xi^{2} A\right)}\left(\frac{z^{2}}{12 \xi^{2} A}(6 \xi-1)+6 y(2-3 c \xi)+3\left(1-\frac{z^{2}}{72 \xi^{2} A}-y-z\right)(1-3 \omega)\right)-2\right)+y z, \\
z^{\prime}= & \left(-3 z-\frac{6}{1+18 \xi^{2} A}\left(\frac{z^{2}}{12}(6 \xi-1)+y \xi(12 \xi A+c)+3 \xi^{2} A\left(1-\frac{z^{2}}{72 \xi^{2} A}-y-z\right)(1-3 \omega)\right)\right) \\
& +\frac{z^{2}}{6 \xi A}-z\left(\frac{1}{6\left(1+18 \xi^{2} A\right)}\left(\frac{z^{2}}{12 \xi^{2} A}(6 \xi-1)+6 y(2-3 c \xi)+3\left(1-\frac{z^{2}}{72 \xi^{2} A}-y-z\right)(1-3 \omega)\right)-2\right)+z^{2}, \\
A^{\prime}= & \frac{z}{3 \xi}+A z .
\end{aligned}
$$

We enumerate the stationary points and corresponding solutions of (68),

\section{1. de-Sitter solution}

$$
\begin{aligned}
y & =1, z=0, A=-\frac{c}{12 \xi}, \Omega=0, \\
Y & =12, \beta=0, \\
a(t) & =a_{0} e^{H_{0}\left(t-t_{0}\right)}, \\
\phi(t) & =\phi_{0},
\end{aligned}
$$

where $\phi_{0}= \pm \sqrt{\frac{n}{6 \xi(n-4)}}, H_{0}= \pm \sqrt{-\frac{V_{0} n \phi_{0} n^{-2}}{12 \xi}}$ are obtained from the system (3)- (5) for $\dot{H}_{0}=\dot{\phi}_{0}=\ddot{\phi}_{0}=\rho=0$. This is de Sitter solution which exists also for $N>2$. We have not solved the autonomous system in this case as the combination $4 x+z^{2}$ appearing in the denominator vanishes in case of de Sitter solution with coordinates $x=0$, $y=1, z=0, A=-\frac{n}{12 \xi}, \Omega=0$. 
2.

$$
\begin{aligned}
y & =0, z=12 \xi+2 \sqrt{36 \xi^{2}-6 \xi}, \\
A & =-\frac{1}{3 \xi}, \Omega=0 \\
Y & =6(12 \xi-1+2 \sqrt{6 \xi(6 \xi-1)}), \\
\beta & =-6 \xi-\sqrt{6 \xi(6 \xi-1)}, \\
a(t) & =a_{0}\left|t-t_{0}\right|^{\frac{1}{3-12 \xi-2 \sqrt{6 \xi(6 \xi-1)}}}, \\
\phi(t) & =\phi_{0}\left|t-t_{0}\right|^{-\frac{6 \xi+\sqrt{6 \xi(6 \xi-1)}}{3-12 \xi-2 \sqrt{6 \xi(6 \xi-1)}}},
\end{aligned}
$$

3.

$$
\begin{aligned}
y & =0, z=12 \xi-2 \sqrt{36 \xi^{2}-6 \xi}, \\
A & =-\frac{1}{3 \xi}, \Omega=0, \\
Y & =6(12 \xi-1-2 \sqrt{6 \xi(6 \xi-1)}), \\
\beta & =-6 \xi+\sqrt{6 \xi(6 \xi-1)}, \\
a(t) & =a_{0}\left|t-t_{0}\right|^{\frac{1}{3-12 \xi+2 \sqrt{6 \xi(6 \xi-1)}}}, \\
\phi(t) & =\phi_{0}\left|t-t_{0}\right|^{\frac{-6 \xi+\sqrt{6 \xi(6 \xi-1)}}{3-12 \xi+2 \sqrt{6 \xi(6 \xi-1)}}},
\end{aligned}
$$

Solutions corresponding to stationary points 2,3 were found in [16], where the cosmological model with non-minimal coupling $F(\phi)=\xi \phi^{2}$ and the effective potential $V(\phi)=\lambda \phi^{n}$ (without Einstein term in the Lagrangian) was investigated.

4.

$$
\begin{aligned}
y & =0, z=-\frac{4 \xi(1-3 \omega)}{4 \xi+\omega-1}, A=-\frac{1}{3 \xi}, \\
\Omega & =1+\frac{2 \xi(1-3 \omega)^{2}}{3(4 \xi+\omega-1)^{2}}+\frac{4 \xi(1-3 \omega)}{4 \xi+\omega-1}, \\
Y & =\frac{3(1-3 \omega)(\omega-1)}{4 \xi+\omega-1}, \beta=\frac{2 \xi(1-3 \omega)}{4 \xi+\omega-1}, \\
a(t) & =a_{0}\left|t-t_{0}\right|^{\frac{2(4 \xi+\omega-1)}{3 \omega^{2}+16 \xi-3}}, \\
\phi(t) & =\phi_{0}\left|t-t_{0}\right|^{\frac{4 \xi(1-3 \omega)}{3 \omega^{2}+16 \xi-3}},
\end{aligned}
$$

This solution also was found in [16].

5.

$$
\begin{aligned}
y & =-\frac{\left(6 \xi \omega(c-2)-2 \xi(c+6)+3-3 \omega^{2}\right)}{2 c^{2} \xi} \\
z & =\frac{6(1+\omega)}{c}, A=-\frac{1}{3 \xi} \\
\Omega & =\frac{3(1-\xi(c+2)) \omega+c^{2} \xi+3-7 c \xi-6 \xi}{c^{2} \xi} \\
Y & =\frac{3(6+c-3 \omega(c-2))}{c}, \beta=-\frac{3(1+\omega)}{c} \\
a(t) & =a_{0}\left|t-t_{0}\right|^{\frac{2 n}{3(n-2)(1+\omega)}} \\
\phi(t) & =\phi_{0}\left|t-t_{0}\right|^{\frac{2}{2-n}} \\
\rho(t) & =\rho_{0}\left|t-t_{0}\right|^{\frac{2 n}{2-n}}
\end{aligned}
$$


We note that the analogous solution exists in the case $N>2$ (see (34)). It also was obtained in [16]. For $n=2$ the power index of functions $a(t)$ and $\phi(t)$ becomes infinity and power-law takes the exponential form. In this case the coordinates of the point 5 are $y=\frac{16 \xi-3+3 \omega^{2}}{8 \xi}, z=3(\omega+1), A=-\frac{1}{3 \xi}, \Omega=\frac{3(1-4 \xi) \omega-16 \xi+3}{4 \xi}$ and also, $Y=12$, $\beta=-\frac{3(1+\omega)}{2}$. This means that, $\frac{\dot{H}}{H^{2}}=0, H=H_{0}=$ const, $\frac{\dot{\phi}}{\phi}=\beta H_{0}$ giving rise to the following

$$
\begin{aligned}
& a(t)=a_{0} e^{H_{0}\left(t-t_{0}\right)}, \\
& \phi(t)=\phi_{0} e^{\beta H_{0}\left(t-t_{0}\right)}=\phi_{0} e^{-\frac{3 H_{0}(1+\omega)\left(t-t_{0}\right)}{2}}, \\
& \rho(t)=\rho_{0} e^{-3 H_{0}(1+\omega)\left(t-t_{0}\right)} .
\end{aligned}
$$

In order to find the constants $H_{0}$ and $\rho_{0}$, we substitute (74) in definition (10) of coordinates $y, \Omega$ taking into account that $n=2, y=\frac{16 \xi-3+3 \omega^{2}}{8 \xi}$ and $\Omega=\frac{3(1-4 \xi) \omega-16 \xi+3}{4 \xi}$,

$$
\begin{aligned}
y & =\frac{2 V_{0} \phi^{n}}{H^{2}\left(1-6 \xi \phi^{2}\right)}=\frac{2 V_{0} \phi_{0}{ }^{2} e^{-3 H_{0}(1+\omega)\left(t-t_{0}\right)}}{H_{0}{ }^{2}\left(1-6 \xi \phi_{0}{ }^{2} e^{-3 H_{0}(1+\omega)\left(t-t_{0}\right)}\right)} \rightarrow-\frac{V_{0}}{3 H_{0}{ }^{2} \xi} \\
\Omega & =\frac{2 \rho}{H^{2}\left(1-6 \xi \phi^{2}\right)}=\frac{2 \rho_{0} e^{-3 H_{0}(1+\omega)\left(t-t_{0}\right)}}{{H_{0}}^{2}\left(1-6 \xi \phi_{0}{ }^{2} e^{-3 H_{0}(1+\omega)\left(t-t_{0}\right)}\right)} \rightarrow-\frac{\rho_{0}}{3 H_{0}{ }^{2} \phi_{0}{ }^{2} \xi}
\end{aligned}
$$

for $t \rightarrow t_{0}$ when $H_{0}>0$ (or for $t \rightarrow \infty$ when $\left.H_{0}<0\right)$.

Therefore,

$$
H_{0}^{2}=-\frac{V_{0}}{3 \xi y}=-\frac{8 V_{0}}{3\left(16 \xi-3+3 \omega^{2}\right)}
$$

where $\xi<\frac{3\left(1-\omega^{2}\right)}{16}, V_{0}>0$ or $\xi>\frac{3\left(1-\omega^{2}\right)}{16}, V_{0}<0$ and

$$
\rho_{0}=-3 \Omega H_{0}{ }^{2} \phi_{0}{ }^{2} \xi=\frac{2 V_{0} \phi_{0}{ }^{2}(3(1-4 \xi) \omega-16 \xi+3)}{16 \xi-3+3 \omega^{2}}
$$

6.

$$
\begin{aligned}
y & =-\frac{\left(6 c^{2} \xi^{2}-c^{2} \xi+8 c \xi-48 \xi^{2} c-120 \xi^{2}+56 \xi-6\right)}{6(\xi(2+c)-1)^{2}}, \\
z & =\frac{2 \xi(c-4)}{\xi(2+c)-1}, A=-\frac{1}{3 \xi}, \Omega=0 \\
Y & =\frac{3\left(10 c \xi-c^{2} \xi-4\right)}{\xi(2+c)-1}, \beta=-\frac{\xi(c-4)}{\xi(2+c)-1}, \\
a(t) & =a_{0}\left|t-t_{0}\right|^{\frac{2(\xi(2+n)-1)}{\xi(n-2)(n-4)}} \\
\phi(t) & =\phi_{0}\left|t-t_{0}\right|^{\frac{2}{2-n}}
\end{aligned}
$$

This solution in the limit $\xi \rightarrow \infty$ gives (40) [16].

For $n=2$ and $n=4$ power indexes of functions $a(t)$ and $\phi(t)$ become infinity and power-law solutions transform into exponential ones. We find that coordinates of point 6 in these cases are, $y=\frac{96 \xi^{2}-34 \xi+3}{3(4 \xi-1)^{2}}, z=-\frac{4 \xi}{4 \xi-1}, A=-\frac{1}{3 \xi}, \Omega=0$ and $y=1, z=0, A=-\frac{1}{3 \xi}, \Omega=0$ accordingly for $n=2$ and $n=4$. Then $Y=12, \beta=\frac{2 \xi}{4 \xi-1}-$ for $n=2$ and $Y=12$, $\beta=0-$ for $n=4$. For $n=2$ we obtain $\frac{\dot{H}}{H^{2}}=0, H=H_{0}=$ const and

$$
\begin{aligned}
& a(t)=a_{0} e^{H_{0}\left(t-t_{0}\right)}, \\
& \phi(t)=\phi_{0} e^{\beta H_{0}\left(t-t_{0}\right)}=\phi_{0} e^{\frac{2 H_{0} \xi\left(t-t_{0}\right)}{4 \xi-1}} .
\end{aligned}
$$

Using the definition of the coordinate $y$ from (10) analogous to previous point we find the constant $H_{0}(n=2$, $\left.y=\frac{96 \xi^{2}-34 \xi+3}{3(4 \xi-1)^{2}}\right)$ and hence ${H_{0}}^{2}=-\frac{V_{0}}{3 \xi y}=-\frac{V_{0}(4 \xi-1)^{2}}{\xi\left(96 \xi^{2}-34 \xi+3\right)}$, where $\xi \in(-\infty, 0) \bigcup\left(\frac{1}{6}, \frac{9}{48}\right), V_{0}>0$ or $\xi \in\left(0, \frac{1}{6}\right) \bigcup\left(\frac{9}{48}\right)$, $V_{0}<0$. For $n=4$ we get $\frac{\dot{\phi}}{\phi}=0$ and

$$
\begin{aligned}
& a(t)=a_{0} e^{H_{0}\left(t-t_{0}\right)}=a_{0}, \\
& \phi(t)=\phi_{0}=0,
\end{aligned}
$$


where values $H_{0}=0, \phi_{0}=0$ were found by substitution $a(t)=a_{0} e^{H_{0}\left(t-t_{0}\right)}, \phi(t)=\phi_{0}, n=4$ in the system of equations (3)-(5).

[1] C. Brans and R. Dicke, Phys. Rev. 124, 925 (1961).

[2] F.Bezrukov and M.Shaposhnikov, Phys.Lett. B639, 703 (2008).

[3] S. Perlmutter el al, Astrophysics, J. 157, 565(1999).

[4] A. Reiss et al, Astrophysics J. 117, 707(1999).

[5] V. Sahni and A. A. Starobinsky, Int. J. Mod. Phys. D 9, 373 (2000).

[6] V. Sahni and A. Starobinsky, Int.J.Mod.Phys.D 15, 2105(2006) astro-ph/0610026; T. Padmanabhan, astro-ph/0603114 P. J. E. Peebles and B. Ratra, Rev. Mod. Phys. 75, 559 (2003); L. Perivolaropoulos, astro-ph/0601014 N. Straumann, arXiv:gr-qc/0311083 J. Frieman, arXiv:0904.1832, M. Sami, Lect. Notes Phys.72, 219(2007); M. Sami, arXiv:0901.0756; K. Bamba, S. Capozziello, S. Nojiri and S. D. Odintsov, arXiv:1205.3421 S. Tsujikawa, arXiv:1004.1493.

[7] E. J. Copeland, M. Sami and S. Tsujikawa, Int. J. Mod. Phys., D15 , 1753(2006) hep-th/0603057.

[8] E. V. Linder, Rep. Prog. Phys. 71 (2008) 056901.

[9] Robert R. Caldwell and Marc Kamionkowski arXiv:0903.0866.

[10] A. Silvestri and Mark Trodden, arXiv:0904.0024.

[11] J. Frieman, M. Turner and D. Huterer, arXiv:0803.0982

[12] M. Sami, Curr. Sci. 97,887(2009) arXiv:0905.2284

[13] R.Gannouji, D.Polarski, A.Ranquet and A.Starobinsky, JCAP 0609:016 (2006).

[14] L.Amendola, R.Gannouji, D.Polarski, S.Tsujikawa, Phys. Rev. D75, 083504 (2007).

[15] S.Carloni, A.Troisi, P.Dunsby, Gen.Rel.Grav.41, 1757 (2009).

[16] S.Carloni, J.Leach, S.Capozziello, P.Dunsby. Class. Quant. Grav. 25, 035008 (2008).

[17] M. Szydlowski and O. Hrycyna, JCAP 0901, 039 (2009)[arXiv:0811.1493v2].

[18] E. Elizalde, S. Nojiri, S. D. Odintsov, D. Sez-Gmez and V. Faraoni, Phys.Rev.D77:106005,2008 arXiv:0803.1311.

[19] L.Jarv, P.Kuusk, M.Saal, Phys. Rev. D76: 103506 (2008), Phys. Rev. D78: 083530 (2008), Phys. Rev. D81: 104007 (2010), Phys. Lett. A694, 1 (2010); Gaveshna Gupta, Emmanuel N. Saridakis, and Anjan A. Sen, Phys. Rev. D 79 (2009) 123013; Y. -F. Cai, E. N. Saridakis, M. R. Setare and J. -Q. Xia, Phys. Rept. 493, 1 (2010) arXiv:0909.2776 [hep-th]]; E. Elizalde, E.O. Pozdeeva and S.Yu. Vernov, De Sitter Universe in Non-local Gravity, Phys. Rev. D 85 (2012) 044002, arXiv:1110.5806

[20] A.Yu.Kamenshchik, I.M.Khalatnikov, A.V.Toporensky, Int. J. Mod. Phys. D6 (1997), 649.

[21] Amna Ali, R. Gannouji and M. Sami, Phys. Rev. D 82 (2010) 103015; R. Gannouji and M. Sami, Phys. Rev. D 82 (2010) 024011

[22] V. Sahni, T. D. Saini, A. A. Starobinsky and U. Alam, JETP Lett. 77, 201 (2003); U. Alam, V. Sahni, T. D. Saini and A. A. Starobinsky, Mon. Not. Roy. Ast. Soc. 344, 1057 (2003).

[23] M. Arabsalmani, V. Sahni, Phys. Rev. D 83, 043501 (2011).

[24] B. R. Chang, H. Y. Liu, L. X. Xu, C. W. Zhang and Y. L. Ping, Statefinder parameters for interacting phantom energy with dark matter,JCAP 0701:016 (2007) [astro-ph/0612616].

[25] V. Gorini, A. Kamenshchik and U. Moschella, Can the Chaplygin gas be a plausible model for dark energy? Phys. Rev. D 67, 063509 (2003) [astro-ph/0209395].

[26] X. Zhang, Int. J. Mod. Phys. D 14, 063509 (2005) [astro-ph/0504586]; M. R. Setare and M. Jamil, Gen. Rel. Gravit. 43:293303 (2011) [arXiv:1008.4763].

[27] L. P. Chimento, A. S. Jakubi, D. Pavon and W. Zimdahl, Phys. Rev. D67, 083513 (2003) [astro-ph/0303145]; L. Zhang, J. Cui, J. Zhang, and X. Zhang,,Interacting model of new agegraphic dark energy: Cosmological evolution and statefinder diagnostic, Int. J. Mod. Phys. D19, 21 (2010) [arXiv:0911.2838]; A. Khodam-Mohammadi and M. Malekjani, Cosmic Behavior, Statefinder Diagnostic and $w-w^{\prime}$ Analysis for Interacting NADE model in Non-flat Universe, Astrophys. Space Sci.331:265-273 (2011) [arXiv:1003.0543].

[28] Z. L. Yi and T. J. Zhang, Statefinder diagnostic for the modified polytropic Cardassian universe, Phys. Rev. D75, 083515 (2007) [astro-ph/0703630]; M. Tong, Y. Zhang and T. Xia, Statefinder parameters for quantum effective Yang-Mills condensate dark energy model,Int. J. Mod. Phys. D18, 797 (2009) [arXiv:0809.2123]; H. Farajollahi and A. Salehi, Attractors, Statefinders and Observational Measurement for Chameleonic Brans-Dicke Cosmology,JCAP 1011:006 (2010) [arXiv:1010.3589].

[29] A. Kamenshchik, U. Moschella and V. Pasquier, Phys. Lett. B 511265 (2001).

[30] G. Dvali, G. Gabadadze and M. Porrati, Phys. Lett. B 485, 208 (2000).

[31] V. Faraoni, Phys. Rev. D62, 023504 (2000) [arXiv:0002091v2](2000).

[32] U. Alam and V. Sahni, astro-ph/0209443

[33] Yuri Shtanov and V. Sahni, JCAP 0311, 014 (2003) astro-ph/0202346.

[34] We thank A. Starobinsky for bringing this important result to our notice: A. Starobinsky, Astron. Lett. 7, 36(1981). 